\title{
A Multiresolution Ensemble Hybrid 4DEnVar for Global Numerical Prediction
}

\author{
JUNKYUNG KAY AND XUGUANG WANG \\ School of Meteorology, University of Oklahoma, Norman, Oklahoma
}

(Manuscript received 8 January 2019, in final form 3 November 2019)

\begin{abstract}
A multiresolution ensemble (MR-ENS) method is developed to resolve a wider range of scales of the background error covariance (BEC) in the hybrid four-dimensional ensemble-variational (4DEnVar) while saving computational costs. MR-ENS is implemented in the NCEP Global Forecast System (GFS) gridpoint statistical interpolation (GSI) hybrid 4DEnVar. MR-ENS generates analysis increment by incorporating high-resolution static BEC and flow-dependent ensemble BECs from both high and low resolutions. MR-ENS is compared with three 4DEnVar update approaches: 1) the single-resolution (SR)-Low approach where the analysis increments are generated from the ensemble BEC and the static BEC at the same low resolution; 2) the dual-resolution (DR) approach where the analysis increment is generated using the highresolution static BEC and low-resolution ensemble BEC; and 3) the SR-High approach, which is the same as 1) except that all covariances are at high-resolution. Experiments show that MR-ENS improves global and tropical cyclone track forecasts compared to SR-Low and DR. Inclusion of the high-resolution ensemble leads to increased background ensemble spread, better fitting of the background to observations, increased effective ranks, more accurate ensemble error correlation, and increased power of analysis increment at small scales. The majority of the improvement of MR-ENS relative to SR-Low is due to the partial use of highresolution background ensemble. Compared to SR-High, MR-ENS decreases the overall cost by about $40 \%$ and shows comparable global and tropical cyclone track forecast performances. Diagnostics show that particularly in the tropics, MR-ENS improves the analysis increment over a wide range of scales and increases the effective rank of the ensemble BEC to the degree comparable to SR-High.
\end{abstract}

\section{Introduction}

A hybrid four-dimensional ensemble-variational (4DEnVar) data assimilation system has been implemented for the National Centers for Environmental Prediction (NCEP) Global Forecast System (GFS) model based on the gridpoint statistical interpolation (GSI) system since 2016. Wang and Lei (2014) described the formula of 4DEnVar implemented in GSI and showed that the GFS hybrid 4DEnVar improved the analysis and forecast more than the 3DEnVar (Wang et al. 2013) in both the global and tropical cyclone track forecasts due to the capability of 4DEnVar to account for temporal evolution of the error covariance. Their experiments were conducted through assimilating real operational observations. Kleist and Ide (2015) conducted an observing system simulation experiment (OSSE) and consistently found that moving from the 3D to $4 \mathrm{D}$ ensemble covariance in the hybrid EnVar improves the analysis and forecast for global prediction.

Corresponding author: Xuguang Wang, xuguang.wang@ou.edu
Several recent studies investigate the impact of increasing ensemble size and model resolutions on the analysis and subsequent forecast for GFS hybrid 4DEnVar. Lei and Whitaker (2017) directly increased the ensemble size and resolution in the GFS hybrid 4DEnVar system. They found under the constraints of the same computational cost, increasing ensemble resolution improved the analysis and forecasts more than increasing the ensemble size. This result is consistent with previous studies showing that increasing the ensemble resolution has a larger effect on the performance of short-range ensemble forecasts than increasing the ensemble size (Ma et al. 2012; Raynaud and Bouttier 2017). Huang and Wang (2018) investigated an inexpensive, valid time shifting (VTS) method to increase the ensemble size of the background ensemble in the GFS hybrid 4DEnVar and found this method can improve both global and hurricane track forecast without incurring too much additional cost. In the case of hurricane track forecast, VTS even outperforms the more expensive configuration of directly running more background ensemble members. 
Due to the increasingly available computational resources at operational centers, the spatial resolution of the global model has regularly increased, which contributes to the continued improvement of forecast skills (Hamill et al. 2010). However, to save the computational costs, many operational centers still run the background ensemble of the EnVar data assimilation system or its variant at a reduced resolution compared to the control background (Kleist 2012; Clayton et al. 2013; Lei and Whitaker 2017). This reduced resolution ensemble strategy in the hybrid EnVar has also been adopted for convection allowing hurricane and severe weather data assimilation ( $\mathrm{Lu}$ et al. 2017; Wang and Wang 2019, manuscript submitted to Mon. Wea. Rev.).

The current GSI-based operational 4DEnVar system uses a configuration where the control forecast is at the T1534 horizontal resolution, and the 80 member background ensembles and the static covariance are at the T574 horizontal resolution (Lei and Whitaker 2017). In other words, the analysis increments are generated at a reduced low resolution using the flow-dependent ensemble background error covariance (BEC) and static BEC components that are both at the same low resolution. These low-resolution analysis increments are interpolated onto the high-resolution grid before being added to the high-resolution control forecast. Given the analysis increments contributed by the static and ensemble covariances are both produced at the same reduced low resolution, hereafter we refer to this configuration as single resolution-low (SR-Low). In the meantime, the current GSI-based 4DEnVar has a capability to generate the analysis increment at high resolution by using the high-resolution static BEC and the reduced low-resolution ensemble BEC (Kleist and Ide 2015). Hereafter, we refer this configuration as dual resolution (DR), given the two covariances are at two different resolutions. Although the reduced ensemble approach such as SR-Low and DR can save computational cost, ensemble BEC does not contribute to the highly flow-dependent analysis increment at smaller scales. As a result, useful information in the high-resolution observations is not effectively utilized (Daley 1994).

To mitigate the weakness of using the reduced lowresolution ensemble in the hybrid 4DEnVar, we present a method to still include flow-dependent high-resolution background covariance while not incurring too much additional cost. Rather than using high-resolution for all background ensemble members, we introduce increment terms contributed by the background ensembles at multiple resolutions into the current GSI-based 4DEnVar. In other words, the variational update is performed using high-resolution static BEC, and a mixture of low-resolution ensemble BEC and highresolution ensemble BEC. Hereafter, we refer this method as multiresolution ensemble or (MR-ENS) 4DEnVar. The multiresolution strategy for data assimilation has been investigated using local ensemble transform Kalman filter (LETKF) framework to take background covariance that combines a high-resolution ensemble and a low-resolution ensemble in the idealized Lorenz model (Rainwater and Hunt 2013). This study is the first to explore MR-ENS in the EnVar framework. In this study, we first introduce the MR-ENS formula and its implementation in the GSI 4DEnVar framework. We then investigate MR-ENS in the context of global NWP. The implementation of the MR-ENS approach allows designing a number of experiments to facilitate addressing series of questions on optimal configuration of global 4DEnVar. For this very first study using the newly developed MR-ENS 4DEnVar, experiments are designed and performed to investigate a specific set of questions. Specifically, in addition to the SR-Low and DR experiments, an additional experiment where increments are contributed by both highresolution static and high-resolution ensemble BECs (hereafter denoted as SR-High) is performed. These experiments all have the same number of background ensemble members. They are designed to address the following questions: What is the value of increasing the resolution of part of the background ensemble members through MR-ENS compared to using the coarse resolution for all background ensemble members (MR-ENS versus DR)? To what extent would MR-ENS recover the benefit of more costly approach of using the high resolution for all background ensemble members (MRENS versus SR-High)? Given SR-Low is similar to the operational configuration, how is MR-ENS compared with SR-Low and to what extent their difference is explained by the partial use of the high-resolution background ensemble? Comprehensive diagnostics are performed to facilitate addressing these questions and to understand the differences among these configurations.

Section 2 describes the formula of the newly developed MR-ENS 4DEnVar. Section 3 provides an overview of the experimental design. Comprehensive diagnostics and evaluation are presented in section 4 , and a summary and discussion are provided in section 5 .

\section{Methodology: Multiresolution ensemble hybrid 4DEnVar formulation and implementation}

We first describe the operational GSI 4DEnVar formulation following the notation of Wang (2010) and Wang and Lei (2014). Based on the formulation, a description of the new MR-ENS hybrid 4DEnVar formula 
and its implementation into the GSI minimization is followed. To distinguish the resolution of each component of the formula, index $H$ and $L$ are added to the formulation of the high- and low-resolution background ensemble components, respectively.

\section{a. GSI 4DEnVar formulation}

In the operational GSI hybrid 4DEnVar DA system, the four-dimensional flow-dependent ensemble covariance is incorporated into the GSI by using the augmented control vectors. The minimization of the 4DEnVar cost function is performed with respect to control vectors associated with the static and ensemble covariances. The time evolution of the increment associated with static covariance is ignored in the data assimilation window, while increment associated with the ensemble covariance is propagated effectively through four-dimensional ensemble covariance. The increments at multiple times (denoted with subscript $t$ ) are obtained in the data assimilation window.

The analysis increment $\mathbf{x}_{t}^{\prime}$ is defined as

$$
\mathbf{x}_{t}^{\prime}=\mathbf{u}\left\{\left(\mathbf{x}_{1}^{\prime}\right)_{t}+\sum_{k=1}^{K}\left[\boldsymbol{\alpha}_{k} \circ\left(\mathbf{x}_{k}^{e}\right)_{t}\right]\right\}
$$

where the first term on the right side, $\left(\mathbf{x}_{1}^{\prime}\right)_{t}$, is the increment associated with the GSI static covariance at multiple times $t=1, \ldots, L$ within the data assimilation window. In the current implementation of the operational GSI 4DEnVar, $\left(\mathbf{x}_{1}^{\prime}\right)_{t}$ does not vary within the DA window. The second term, $\sum_{k=1}^{K}\left[\boldsymbol{\alpha}_{k} \circ\left(\mathbf{x}_{k}^{e}\right)_{t}\right]$, is the increment associated with the flow-dependent ensemble covariance at time $t .\left(\mathbf{x}_{k}^{e}\right)_{t}$ denotes the $k$ th ensemble perturbation at time $t$ normalized by $\sqrt{K-1}$, and $K$ is the ensemble size. The vector $\boldsymbol{\alpha}_{k}$ is the $k$ th augmented control vector. The symbol $\circ$ denotes the Schur product which is an element-by-element product of two matrices (Houtekamer and Mitchell 2001). When all components are at the same resolution, the linear interpolation matrix $\mathbf{u}$ is an identity matrix. In the SR-Low, because $\mathbf{x}_{k}^{e}$ and $\left(\mathbf{x}_{1}^{\prime}\right)_{t}$ have lower resolution than the control forecast, final analysis increment is multiplied by the linear transform matrix $\mathbf{u}$, which interpolates lowresolution grids $\left(m_{L}\right)$ to high-resolution grids $\left(m_{H}\right)$. In the case of DR, while $\left(\mathbf{x}_{1}^{\prime}\right)_{t}$ has the same resolution as the control forecast, $\mathbf{x}_{k}^{e}$ has a lower resolution than the control forecast, so $\mathbf{u}$ is applied to the second term on the right of Eq. (1) to interpolate the increment associated with the ensemble covariance into the high-resolution grids following

$$
\mathbf{x}_{t}^{\prime}=\left(\mathbf{x}_{1}^{\prime}\right)_{t}+\mathbf{u} \sum_{k=1}^{K}\left[\boldsymbol{\alpha}_{k}^{\circ}\left(\mathbf{x}_{k}^{e}\right)_{t}\right] .
$$

Similar DR equation is discussed in Kleist and Ide (2015). In the current implementation of GSI hybrid 4DEnVar, the same $\boldsymbol{\alpha}_{k}$, is applied to the all variables at all time $t$. The analysis increment $\mathbf{x}_{t}^{\prime}$ in Eq. (1) is generated via minimizing the following incremental cost function:

$$
\begin{aligned}
J\left[\left(\mathbf{x}_{1}^{\prime}\right)_{t}, \boldsymbol{\alpha}\right]= & \beta_{1} J_{1}+\beta_{e} J_{e}+J_{o} \\
= & \frac{1}{2} \beta_{1}\left(\mathbf{x}_{1}^{\prime}\right)_{t}^{\mathrm{T}} \mathbf{B}_{1}^{-1}\left(\mathbf{x}_{1}^{\prime}\right)_{t}^{\mathrm{T}}+\frac{1}{2} \beta_{e}(\boldsymbol{\alpha})^{\mathrm{T}} \mathbf{A}^{-1}(\boldsymbol{\alpha}) \\
& +\frac{1}{2} \sum_{t=1}^{L}\left(\mathbf{H} \mathbf{x}_{t}^{\prime}-\mathbf{y}_{t}^{o}\right)^{\mathrm{T}} \mathbf{R}^{-1}\left(\mathbf{H} \mathbf{x}_{t}^{\prime}-\mathbf{y}_{t}^{o}\right) .
\end{aligned}
$$

The minimization of the cost function is based on the full background error covariance preconditioning. In this cost function, the quantity $\mathbf{B}_{1}$ in the first term on the right-hand side denotes the traditional GSI 3DVar BEC associated with the increment $\left(\mathbf{x}_{1}^{\prime}\right)_{t}$. The timeindependent $\mathbf{B}_{1}$ is used at multiple times $t=1, \ldots, L$ within the data assimilation window. In the second term, $\boldsymbol{\alpha}$ is a vector constructed by concatenating vector $\boldsymbol{\alpha}_{k}$ from $k=1$ to $K$, and is constrained by a block-diagonal matrix $\mathbf{A}$ which defines the correlation matrix to localize the ensemble covariance (Lorenc 2003; Wang et al. 2007). The matrix $\mathbf{A}$ is composed of two parts including horizontal covariance localization $\left(\mathbf{A}_{h}\right)$ and vertical covariance localization $\left(\mathbf{A}_{v}\right)$. For the horizontal covariance localization, $\mathbf{A}_{h}$, is predefined as a Gaussian function in a spectral space, and the horizontal localization distance is a function of the model vertical level (Wang and Lei 2014; Kleist and Ide 2015; Lei and Whitaker 2017; Huang and Wang 2018). Vertical covariance localization, $\mathbf{A}_{v}$, is achieved by applying recursive filter transform (Hayden and Purser 1995). The weighting function $\beta_{1}$ and $\beta_{e}$ are constrained to be $\left(1 / \beta_{1}\right)+$ $\left(1 / \beta_{e}\right)=1$ (Wang et al. 2013). The third term defines the observation penalty which denotes the difference between the observation and forecast at time level $t$; $\mathbf{H}$ is the linearized observation operator, $\mathbf{R}$ is the observation error covariance, and $\mathbf{y}_{t}^{o}$ is the observation innovation (observation minus background forecast) at time level $t$.

\section{b. Multiresolution ensemble (MR-ENS) 4DEnVar formulation and implementation}

Because the current study focuses on the impact of the background ensemble in the hybrid DA system, the time index associated with the 4D extension of the ensemble covariance in the 4DEnVar is ignored in this section to simplify the equations. Although all equations look like following the GSI 3DEnVar described in Wang (2010) and Wang et al. (2013), it should be noted 
that the temporal extension is implicitly involved in the equation so that the observation and background at multiple times within the assimilation window are incorporated in 4DEnVar following Wang and Lei (2014).

Analysis increment of MR-ENS 4DEnVar is defined as

$$
\mathbf{x}^{\prime}=\mathbf{x}_{1}^{\prime}+\mathbf{u} \sum_{k=1}^{K_{L}}\left(\boldsymbol{\alpha}_{k}^{L} \circ \mathbf{x}_{k}^{e L}\right)+\sum_{k=1}^{K_{H}}\left(\boldsymbol{\alpha}_{k}^{H} \circ \mathbf{x}_{k}^{e H}\right),
$$

where $\mathbf{u}$ is linear transform matrix interpolating from low resolution to high-resolution grids $\left(m_{H} \times m_{L}\right)$; $\mathbf{x}_{k}^{e L}$ and $\mathbf{x}_{k}^{e H}$ denote the $k$ th low- and high-resolution ensemble perturbations normalized by $\sqrt{K_{L}-1}$ and $\sqrt{K_{H}-1}$, where $K_{L}$ and $K_{H}$ are the low- and highresolution ensemble size, respectively; and $\boldsymbol{\alpha}_{k}^{L}$ and $\boldsymbol{\alpha}_{k}^{H}$ contain the low-resolution and high-resolution extended control vectors, respectively.

By minimizing the following hybrid cost function, we obtain the analysis increment.

$$
\begin{aligned}
J\left(\mathbf{x}_{1}^{\prime}, \boldsymbol{\alpha}^{L}, \boldsymbol{\alpha}^{H}\right)= & \beta_{1} J_{1}+\beta_{L} J_{e}^{L}+\beta_{H} J_{e}^{H}+J_{o} \\
= & \frac{1}{2} \beta_{1}\left(\mathbf{x}_{1}^{\prime}\right)^{\mathrm{T}} \mathbf{B}_{1}^{-1}\left(\mathbf{x}_{1}^{\prime}\right)+\frac{1}{2} \beta_{L}\left(\boldsymbol{\alpha}^{L}\right)^{\mathrm{T}} \mathbf{A}_{L}^{-1}\left(\boldsymbol{\alpha}^{L}\right) \\
& +\frac{1}{2} \beta_{H}\left(\boldsymbol{\alpha}^{H}\right)^{\mathrm{T}} \mathbf{A}_{H}^{-1}\left(\boldsymbol{\alpha}^{H}\right)+\frac{1}{2}\left(\mathbf{H} \mathbf{x}^{\prime}-\mathbf{y}^{o}\right)^{\mathrm{T}} \mathbf{R}^{-1} \\
& \times\left(\mathbf{H} \mathbf{x}^{\prime}-\mathbf{y}^{o}\right) .
\end{aligned}
$$

In Eqs. (4) and (5), $\boldsymbol{\alpha}^{L}$ and $\boldsymbol{\alpha}^{H}$ are vectors formed by concatenating $K_{L}$ and $K_{H}$ vectors $\boldsymbol{\alpha}_{k}^{L}$ and $\boldsymbol{\alpha}_{k}^{H}, k=1, \ldots$, $K_{L}$ and $k=1, \ldots, K_{H}$, respectively. Here $\beta_{L}$ and $\beta_{H}$ are the weighting functions for the low-resolution ensemble and the high-resolution ensemble, respectively, and $\beta_{1}, \beta_{L}$, and $\beta_{H}$ are constrained to be $\left(1 / \beta_{1}\right)+\left(1 / \beta_{L}\right)+$ $\left(1 / \beta_{H}\right)=1$ following Wang et al. (2007).

\section{Experimental design}

The current operational GSI 4DEnVar system for GFS uses a control forecast of T1534 resolution with 64 vertical levels and an 80 ensemble member of T574 resolution with 64 vertical levels. In this study, we use the system with the same configuration as the operational GFS GSI 4DEnVar system except for a reduced resolution. The control forecast is at T670 and ensemble member is at T254 or T670. Similar reduced resolution configuration is used in Lei and Whitaker (2017) and Huang and Wang (2018).

All data assimilation cycling experiments were performed during a 5-week period from 0000 UTC 24 July to 1800 UTC 31 August 2013, and the first 7 days were not used for the verification due to the spinup. This Northern
Hemisphere summer period was chosen so that both the global forecasts and tropical cyclone forecasts can be evaluated. The experiment period is limited to 5 weeks due to the computational constraint. The observation used for assimilation are from the in situ and satellite operational data stream from NCEP global data assimilation system. ${ }^{1}$

To address the gain of using multiple-resolution background ensembles compared to using purely lowor purely high-resolution background ensemble in the hybrid 4DEnVar, the following four experiments are designed. SR-Low is the baseline experiment similarly configured as the operational GFS GSI 4DEnVar. The comparison between MR-ENS and DR reveals how increasing the resolution of part of the background ensemble members will impact the analysis and subsequent forecast. MR-ENS is further compared with SR-High to measure the degree to which MR-ENS can recover the performance of SR-High while saving the computational cost. As discussed later, the difference between MR-ENS and SR-Low is a combined effect of the difference between SR-Low and DR and the difference between MR-ENS and DR. Although not the primary focus, the comparison between SR-Low and DR facilitates the understanding of how much of the difference between MR-ENS and SR-Low is explained by the high-resolution ensemble background. The experiment configuration for all 4 experiments is summarized in Figs. 1 and 2 and Table 1.

\section{a. The single low resolution (SR-Low) experiment}

The DA procedure of the SR-Low approach is illustrated in the upper panel of Fig. 1 and in Fig. 2a. In the SR-Low experiments, the high-resolution background control forecast (T670) is first interpolated to the lowresolution control forecast (T254) to be used as the background of 4DEnVar. It is noted that SR-Low calculates the innovation using the low-resolution backgrounds rather than high-resolution backgrounds for computational efficiency. As shown in Eq. (1) and proven by Wang et al. 2007, the total increment is equivalent to the solution from minimizing a cost function where the low-resolution (T254) static BEC and the low-resolution (T254) flow-dependent BEC calculated by the 80 ensemble forecasts are linearly combined. At the end of the minimization, the low-resolution (T254) increment is interpolated to the high resolution (T670) before being added to the high-resolution (T670) control forecast. The term "single resolution" means that the increments

\footnotetext{
${ }^{1} \mathrm{http} / / /$ www.emc.ncep.noaa.gov/mmb/data_processing/prepbufr.doc/ table_2.htm.
} 


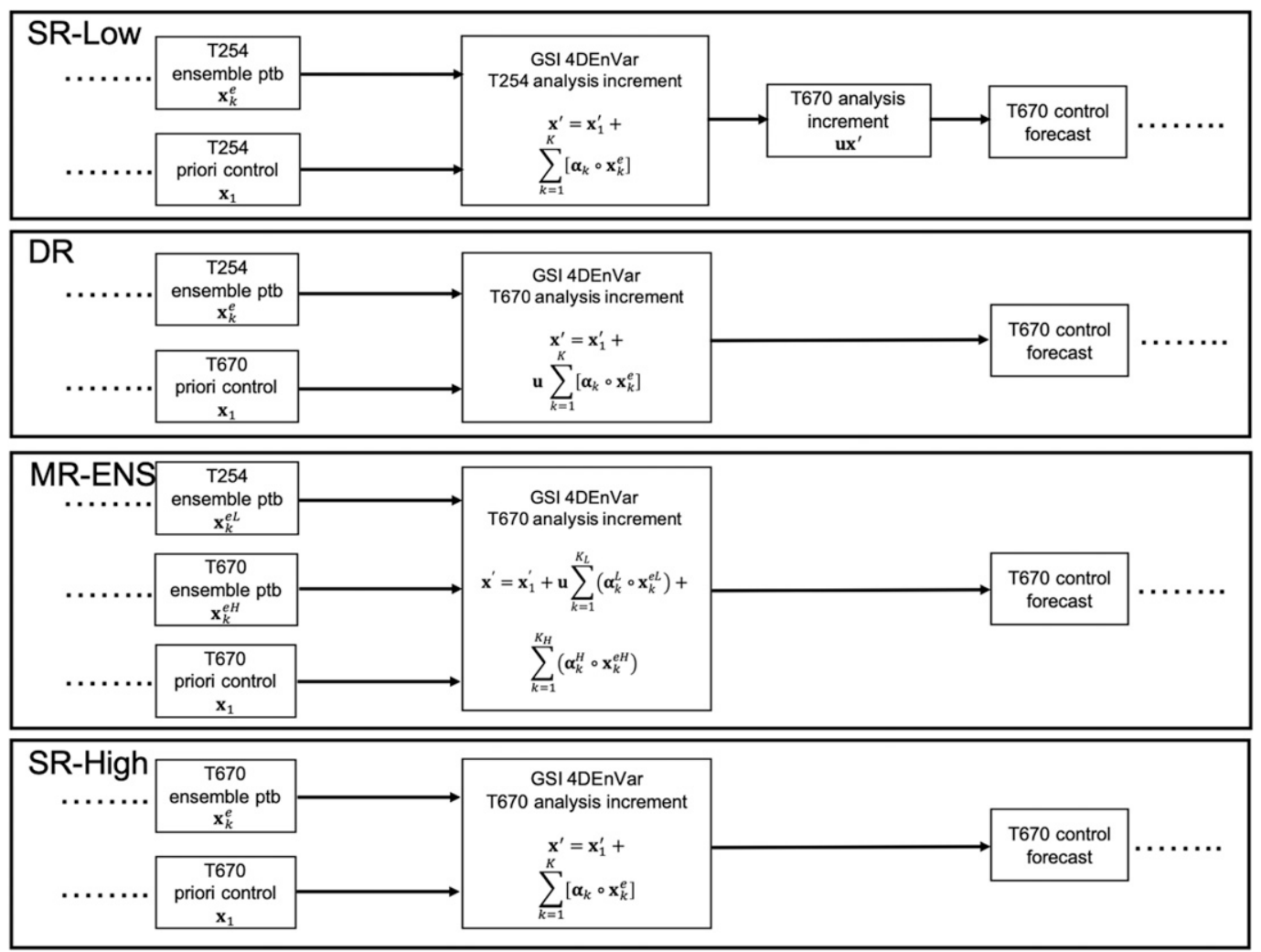

FIG. 1. Flowchart of SR-Low, DR, MR-ENS, and SR-High 4DEnVar data assimilation cycling.

contributed by different sources of BECs are at the single, same resolution. The resolution configuration of each component of the experiment is summarized in Table 1.

The specific settings of the SR-Low experiment are as follows. A weight of $12.5 \%$ on the static BEC and a weight of $87.5 \%$ on the ensemble covariance are used in Eq. (3) following Lei and Whitaker (2017) and Huang and Wang (2018). Lei and Whitaker (2017) found these weights were optimal in global forecast context for an experiment configured similarly as SR-Low. An ensemble Kalman filter (EnKF) with 80 members is adopted to update the ensemble perturbations at the low resolution (T254). Following the two-way coupling method (Wang et al. 2013; Huang and Wang 2018), the updated EnKF ensemble analyses are recentered on the 4DEnVar control analysis that is interpolated from T670 to T254. The ensemble size of 80 is selected following the operational GSI 4DEnVar system (Wang et al. 2013; Kleist and Ide 2015). Hourly ensemble perturbations were used for the 4DEnVar analysis. To suppress the sampling errors in the EnKF, covariance localization was applied using the Gaspari and Cohn localization function (Gaspari and Cohn 1999). We follow the height-dependent horizontal localization scales from Kleist and Ide (2015), Lei and Whitaker
(2017) and Huang and Wang (2018). The horizontal $e$-folding distance is set to 350,1000 , and $1300 \mathrm{~km}$ at levels below $300 \mathrm{hPa}$, between 56 , and $14 \mathrm{hPa}$, and between $5 \mathrm{hPa}$ and the model top, respectively. A constant vertical localization $e$-folding distance of 0.5 scale height was used following these early studies. To further alleviate the deficiency of the system error, the multiplicative inflation was performed by relaxing the posterior ensemble spread to be $85 \%$ of the prior ensemble spread (Whitaker and Hamill 2012); various stochastic physical perturbation schemes were also applied including the stochastically perturbed physics tendencies (SPPT) (Palmer et al. 2009), the stochastic kinetic energy backscatter (SKEB) (Berner et al. 2009), and the stochastically perturbed boundary layer humidity (SHUM) (Tompkins and Berner 2008). A four-dimensional incremental analysis update (4DIAU) method was adopted in the forecast step to reduce the imbalance in the analysis (Lei and Whitaker 2017). Five-day control forecast was run for the evaluation of the global forecast and the tropical cyclone track forecast.

The SR-Low method is computationally inexpensive because it does not require the high-resolution ensemble forecast, and the variational minimization is done fully 
(a) SR-Low

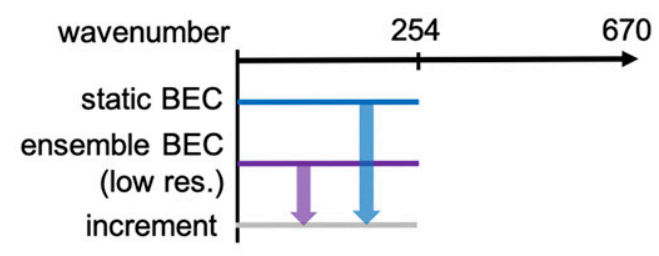

\section{(c) MR-ENS}

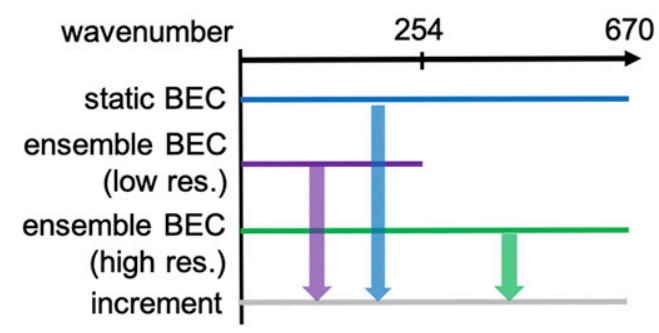

(b) DR

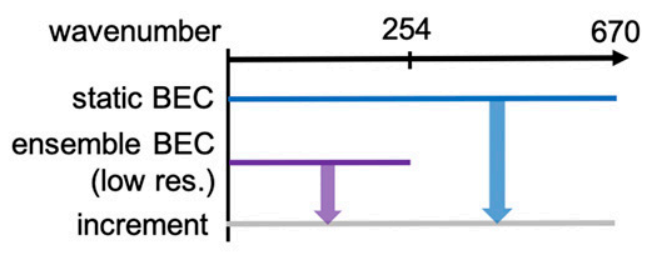

\section{(d) SR-High}

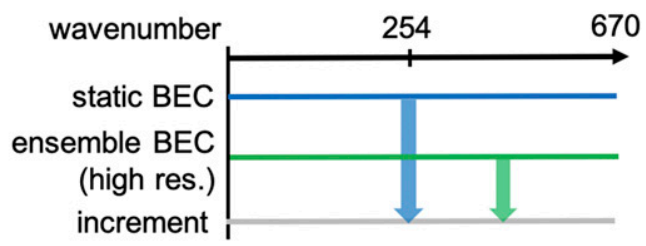

FIG. 2. Schematics of contributions from the static and ensemble background error covariances to the analysis increment in 4DEnVar for (a) SR-Low, (b) DR, (c) MR-ENS, and (d) SR-High. Black, blue, purple, green, and gray lines represent scales, static component, low-resolution ensemble, high-resolution ensemble, and the analysis increment, respectively, in a spectral space.

at the low resolution (T254). This option is therefore currently used in the operational GFS 4DEnVar system. However, the analysis increment does not include any information at the smaller scales beyond the truncation number of the low-resolution ensemble (T254) (Figs. 1 and $2 \mathrm{a}$ ). In addition, from the observation representativeness error point of view (Janjić et al. 2018), the interpolated low-resolution background tends to have more mismatch with those point observations.

\section{b. The dual resolution (DR) experiment}

The DA procedure of the DR approach is illustrated in Figs. 1 and 2b. The term, dual resolution, means that the increments contributed by the static covariance and the ensemble covariance are at two different resolutions. Similar to SR-Low, the analysis increment contributed by the flow-dependent ensemble covariance is at the low resolution (T254). In contrast to SR-Low, the control background during the variational minimization and the static BEC are both at the high resolution (T670). As shown in Eq. (2) and Fig. 1, the low-resolution flowdependent increment is interpolated to T670 before combined with the high-resolution static increment during the variational minimization. DR also does not use the high-resolution ensemble forecast, which saves computational cost. Compared to SR-Low, the expected advantages of DR are as follows. Although the interpolated low-resolution flow-dependent increment does not provide meaningful flow-dependent error covariance at smaller scales, the high-resolution control background and high-resolution static BEC generate high-resolution analysis increment based on

TABLE 1. The configurations of all experiments in terms of control background (ctl back) resolution, ensemble background (ens back) resolution, 4DEnVar analysis increment (ctl anl inc) resolution, control forecast (ctl fcst) resolution, ensemble size, and weighting values on the static and ensemble background error covariances.

\begin{tabular}{|c|c|c|c|c|c|c|}
\hline & \multicolumn{4}{|c|}{ Resolution of 4DEnVar component } & \multirow{3}{*}{$\begin{array}{c}\begin{array}{c}\text { Background } \\
\text { ensemble size }\end{array} \\
\text { T254/T670 }\end{array}$} & \multirow{3}{*}{$\frac{\text { Weighting on BEC }}{\text { Static/ensemble }}$} \\
\hline & \multicolumn{2}{|c|}{$\begin{array}{l}\text { Background for } \\
\text { 4DEnVar }\end{array}$} & \multirow{2}{*}{$\frac{\text { Analysis }}{\text { Ctl anl inc in 4DEnVar }}$} & \multirow{2}{*}{$\frac{\text { Cntl FCST }}{\text { Ctl fcst }}$} & & \\
\hline & Ctl back & Ens back & & & & \\
\hline SR-Low & $\mathrm{T} 254$ & $\mathrm{~T} 254$ & $\mathrm{~T} 254$ & T670 & $80 / 0$ & $0.125 / 0.875$ \\
\hline DR & T670 & $\mathrm{T} 254$ & T670 & T670 & $80 / 0$ & $0.125 / 0.875$ \\
\hline MR-ENS & $\mathrm{T} 670$ & $\mathrm{~T} 254 \mathrm{~T} 670$ & T670 & T670 & $40 / 40$ & $0.125 / 0.4375 / 0.4375$ \\
\hline SR-High & T670 & T670 & T670 & T670 & $0 / 80$ & $0.125 / 0.875$ \\
\hline
\end{tabular}


the climatological error statistics (Fig. 2b). Therefore, from the filtering effect of data assimilation (Daley 1994), DR is able to extract information from the highresolution observations whereas SR-Low cannot. In the process of constructing the observation term (Jo), the innovation of DR is calculated directly from the high-resolution background. SR-Low, in contrast, interpolates the high-resolution background to T254, and therefore the high-resolution background does not involve the minimization. On the other hand, DR involves interpolation of the low-resolution flow-dependent increments to the high-resolution grid during every iteration of the minimization, which can produce spurious increment at small scales due to aliasing (Kleist 2012). This interpolation error accumulated during the iteration can play a negative impact on the analysis. Therefore, the relative performance of DR relative to SR-Low remains to be determined. The rest of the experiment design for DR is the same as SR-Low.

\section{c. The multiresolution ensemble (MR-ENS) experiment}

In the MR-ENS method, the only difference relative to DR is that the flow-dependent increments are contributed from ensemble covariances at multiple resolutions (section 2b). In this study, ensemble covariances with two different resolutions are adopted. It is noted that MR-ENS creates the ensemble perturbations that are fed into the 4DEnVar separately for the high- and lowresolution background ensemble members. Specifically, the 40 low resolution perturbations are calculated as the deviation of each member from the mean of the 40 lowresolution members and the 40 high-resolution perturbations are calculated as the deviation of each member from the mean of the 40 high-resolution members. On the other hand, for all other experiments, given the background members are at the same resolution, the perturbations are calculated from the deviation from the mean of 80 members. As shown in Eq. (4), the total increment of MR-ENS is a linear combination of the high-resolution increment contributed by the static covariance, the highresolution flow-dependent increment directly contributed by the high-resolution background ensemble and the low-resolution increment associated with the lowresolution ensemble. The low-resolution ensemble increment is interpolated to the high resolution before added to the total increment during the minimization. In other words, compared to DR, MR-ENS has an additional advantage of incorporating more realistic highresolution ensemble BEC in the cost function. On the other hand, MR-ENS results in more expensive computational costs compared to DR and SR-Low. As stated in section 1, given SR-Low is mimicking the operational configuration, MR-ENS is additionally compared with SR-Low. The methodological differences described in sections $3 a-3 c$ demonstrate the differences between MR-ENS and SR-Low are a combined effect of the difference between SR-Low and DR and the difference between MR-ENS and DR. Although the focus of the study is not to perform a detailed comparison between SR-Low and DR, their comparison serves to facilitate the understanding of MR-ENS and SR-Low.

As a first step of exploring MR-ENS relative to SRLow, DR, and SR-High, the same number (40) of high (T670) and low (T254) resolution ensemble members are adopted. EnKF is used to update the background ensemble of MR-ENS at the low-resolution (T254). Before the EnKF update, MR-ENS interpolates the highresolution background ensemble components (T670) to the low-resolution (T254). EnKF then updates the whole 80 low resolution background ensembles at the lowresolution (T254) as SR-Low and DR. The updated ensemble members are recentered on the 4DEnVar control analysis that is interpolated from T670 to T254. Half the members of the recentered analysis ensemble are interpolated back to their original high-resolution (T670) for initializing high-resolution ensemble forecasts. Future studies will explore the impact of varying ensemble size between high- and low-resolution ensembles. Lei and Whitaker (2017) found that the optimal value of weight is not sensitive to the ensemble resolution in terms of the global forecast performance. Therefore, as in SR-Low and DR, a weight of $12.5 \%$ is placed on the static BEC, a total weight of $87.5 \%$ is placed on the ensemble covariance with an equal split $(43.75 \%)$ on the low-resolution ensemble BEC and the high-resolution ensemble BEC, respectively. The same covariance localization cutoff radii for low- and high-resolution ensemble as SR-Low are used. Future studies will explore the impact of varying ensemble size, varying weights, and localizations for the high and low-resolution ensembles in MR-ENS. The rest of the setting of the MR-ENS experiment is the same as SR-Low experiment.

\section{d. The single-high-resolution (SR-High) experiment}

The SR-High experiment generally follows the same setup as SR-Low except that all covariances, and increments are produced at high resolution (Figs. 1 and 2d). In other words, SR-High does not need to apply the linear transform matrix, $\mathbf{u}$, to the right side of Eq. (1). Specifically, the total increment is formed as the linear combination of the increments contributed by the high-resolution (T670) static BEC and by the high-resolution flow-dependent BEC estimated by the 80 high-resolution ensemble forecasts (T670). SR-High 

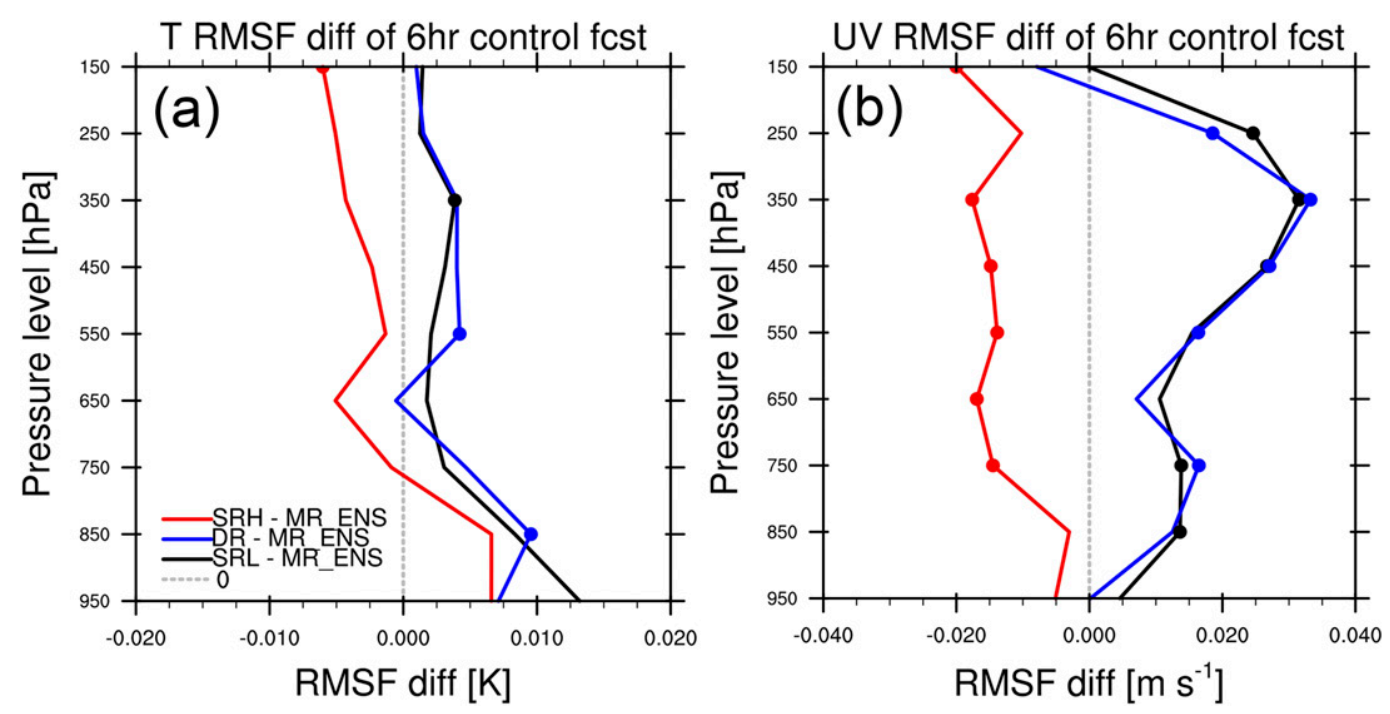

FIG. 3. The globally averaged differences of the rms fit of the 6 -h forecast to the rawinsonde observations between SR-Low and MR-ENS (black line), DR and MR-ENS (blue line), and SR-High and MR-ENS (red line) for (a) temperature and (b) wind as a function of pressure. The dots filled with the corresponding color indicate the level where MR-ENS experiment statistically significantly differ from the other experiments at or above the $95 \%$ confidence level by applying the paired $t$ test.

has the same weighting configuration as SR-Low following Lei and Whitaker (2017), The specific settings of the SR-High experiment are the same as the SRLow experiment except the horizontal localization is further tuned following Lei and Whitaker (2017). Specifically, the localization radius for SR-high measured in terms of the $e$-folding distance is reduced from 350 to $264 \mathrm{~km}$ below $300 \mathrm{hPa}$. In SR-High, for a more fair comparison to the MR-ENS, the EnKF update is done at T254 after interpolating all background ensemble members from T670 to T254. Like MR-ENS, the updated ensemble members are recentered on the 4DEnVar control analysis that is interpolated from T670 into T254. Then all members of the recentered analysis ensemble are interpolated back to T670 for initializing high-resolution ensemble forecast. Parameters used by all experiments are summarized in Table 1.

\section{Results}

In the following results involving statistical significance test, the paired $t$ test is used. Given the potential temporal correlation of the sample datasets over the testing period, a blind implementation of $t$ test which assumes no correlation of the data may exaggerate the statistical significance (e.g., Wang and Bishop 2003; Wilks 2006). To consider the time correlation, a method by Wilks (2006), Kim and Kim (2014), and Yang and Kim (2017) is adopted where the sample variance in the paired $t$ test is inflated by $\left(1+\rho_{1}\right) /\left(1-\rho_{1}\right)$ where $\rho_{1}$ is the lag- 1 autocorrelation coefficient of the sample. Detailed mathematical formula of the $t$ test with inflated sample variance can be found in Yang and Kim (2017). A significance level of $95 \%$ is adopted.

\section{a. Verification of 6-h forecasts against the rawinsondes}

Figure 3 shows the globally averaged difference of the root-mean-square (RMS) fit of the 6-h forecasts to the rawinsonde observations between MR-ENS and the other methods. Positive values represent the improvement of MR-ENS over the other experiment. A filled circle is marked at the level where the difference is statistically significant at and above the $95 \%$ level.

SR-Low and DR show small differences in terms of 6-h forecast fit to observations for both temperature and wind (Figs. 3a,b), which suggests that the effect of just increasing the resolution of the background and the static BEC is not significant or neutral. Compared to SRLow and DR, MR-ENS improves the fitting of the 6-h forecast to both the temperature and wind observations at almost all pressure levels (Figs. 3a,b). Such an improvement is more statistically significant for the wind than the temperature. For the fit to the temperature, SRHigh does not show statistically significant improvement relative to MR-ENS except above $250 \mathrm{hPa}$. At low levels below $850 \mathrm{hPa}$, MR-ENS even better fits the temperature observation than SR-High though the difference is not statistically significant. SR-High shows a better fit to 
the wind observation than MR-ENS with statistical significance at almost all pressure levels (Fig. 3b). The differences among DR, MR-ENS, and SR-High are more significant for the wind field than the temperature field, which suggests varying ensemble resolution has more impact on the wind field than the temperature field. This result is consistent with previous studies that have shown that the flow-dependent ensemble covariance seems to have a relatively smaller effect on the improvement of the temperature forecast compared to the wind forecast (Whitaker et al. 2008; Wang et al. 2013; Wang and Lei 2014; Huang and Wang 2018). One hypothesis was that the background ensemble for temperature is overall more underdispersive than the wind [Wang et al. (2013), also shown in Fig. 6 of this paper]. Additionally, as shown later in Fig. 6, increasing background ensemble resolution has less impact on the ensemble spread for the temperature field than the wind field.

It is worth noting that the inclusion of the highresolution ensemble covariance results in an insignificant difference in the fitting of the 6-h forecast for both temperature and wind below about $850 \mathrm{hPa}$. Past studies showed that increasing the vertical resolution or further increasing the horizontal resolution in the planetary boundary layer (PBL) in the convection resolving model results in improvement in the estimation of the vertical structure of PBL (Aligo et al. 2009; Baklanov et al. 2011; Johnson et al. 2018). However, in this study, the horizontal resolutions of both high- and low-resolution ensembles are too coarse compared to the cloud-resolving resolution. In this case, a significant improvement from increasing horizontal ensemble resolution is hindered within the PBL given the large-scale forcings are rather similar in all experiments (Steeneveld et al. 2006).

\section{b. Verification of analysis and forecast against the ECMWF analysis}

The RMS errors of the 5-day global forecasts were calculated against the European Centre for MediumRange Weather Forecasts (ECMWF) interim reanalysis (ERA-Interim). The difference of the RMS errors between MR-ENS and the other methods are shown in Fig. 4 where positive (negative) values with red (blue) color represent the improvement (degradation) of MRENS over the other experiment. A black dot is marked when the difference is statistically significant at and above the $95 \%$ level. It is noted that the ERA-Interim data used here has a horizontal resolution of about $80 \mathrm{~km}$ and describes the rather smoothed features of the atmosphere. Given the contribution from the high-resolution BECs on the analysis and the forecast at early forecast lead time is more prominent, we only include forecasts beyond the 1-day lead time for the verification against the ERAInterim analysis.
MR-ENS improves both the temperature and wind forecasts for almost all forecast lead times below $200 \mathrm{hPa}$ compared to SR-Low (Figs. 4a,d). Statistically significant improvements are found at $300-400 \mathrm{hPa}$ for temperature and $300-700 \mathrm{hPa}$ up to 60 -h lead time. MR-ENS shows improvement than DR at almost all levels (Figs. 4b,e) with statistically significant improvements found at $300-400 \mathrm{hPa}$ for temperature and 300-700 hPa for wind up to 96-h lead time. As shown in Figs. 2b and 2c, the only difference between MR-ENS and DR is that part of the MR-ENS ensemble covariance is at a higher resolution. The difference of the RMS forecast errors between MR-ENS and DR (shown in Figs. 4b and 4e) suggests that the inclusion of the high-resolution ensemble covariance can significantly improve the forecasts especially at longer lead times.

Comparing Figs. 4a, 4b, 4d, and 4e indicates that the RMS error difference between SR-Low and DR is mixed with SR-Low having larger error than DR at early lead time and SR-Low having smaller error than DR at later lead time. The difference, however, is not statistically significant at all lead times and at all levels (not shown). As shown in Eqs. (1) and (2), the major advantage of DR relative to SR-Low lies in the higher resolution of the control background and the static BEC. The potential disadvantage of DR relative to SR-Low is the aliasing effect from the interpolation of low-resolution ensemble to the high-resolution grid during the iterative minimization. This result in Fig. 4 shows that the advantage and disadvantage of DR relative to SR-Low balance out and the combined effect is negligible on the forecast errors. This result together with that in Fig. 3 suggest the improvement of MR-ENS relative to SR-Low is mostly attributed to the high-resolution flow-dependent background covariance rather than the high-resolution background or the high-resolution static covariance.

Although SR-High is more expensive than MR-ENS, the difference between MR-ENS and SR-High is not consistent and is statistically insignificant for all forecast lead times and all pressure levels (Figs. 4c,f). These results comparing all four experiments confirm our hypothesis stated in the introduction that increasing the ensemble covariance resolution in 4DEnVar can improve the global forecasts and a cost-effective way such as increasing the resolution of partial background ensemble members could achieve similar improvements as increasing the resolution of all background ensemble members.

\section{c. Tropical cyclone track forecast}

Tropical cyclones (TCs) are usually formed by interaction between small-scale convection and large-scale environment in the early stages, and the track of TCs is dominated by the large-scale steering flow in the TC environment (Evans et al. 2011). In this study, the 


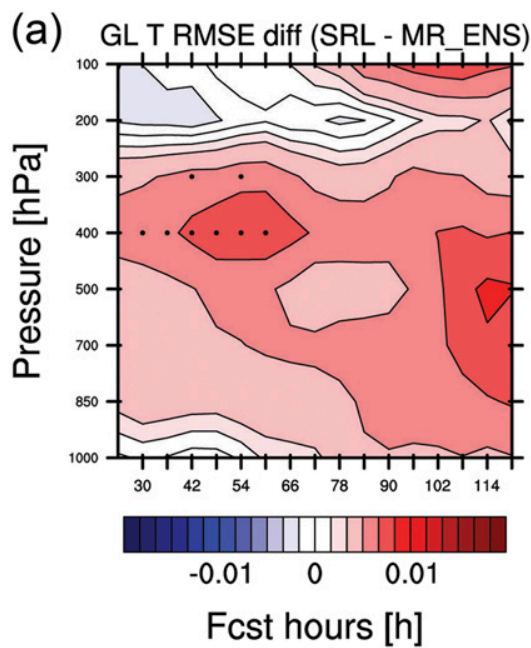

(d) GL UV RMSE diff (SRL - MR ENS)

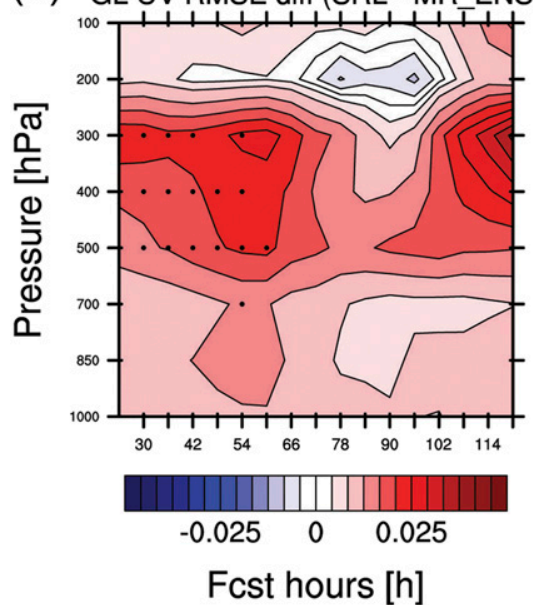

(b)

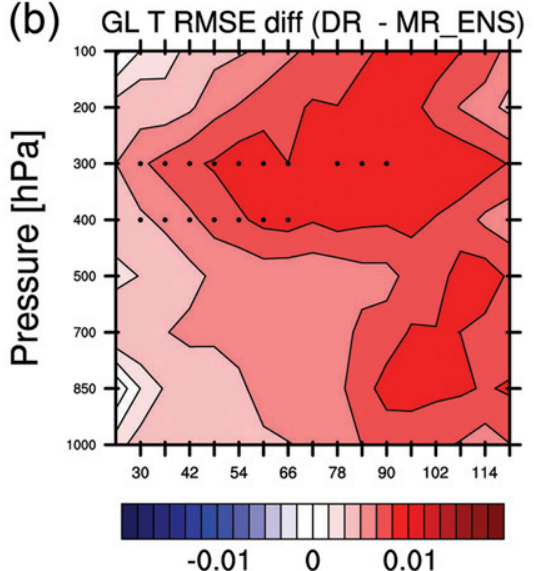

Fcst hours [h]

(e)

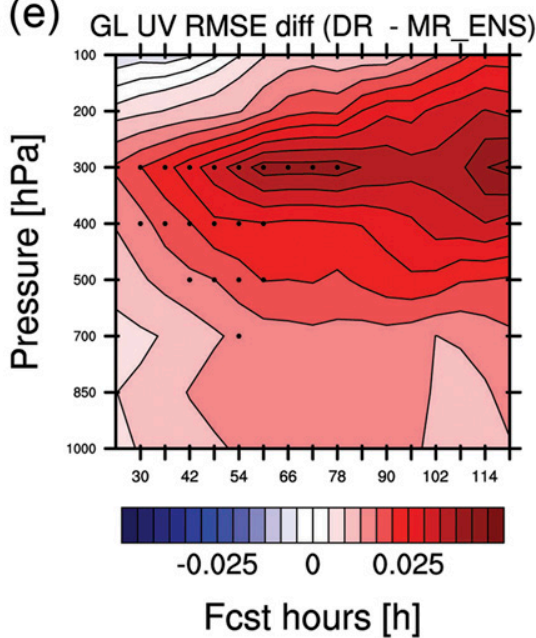

(c) GL T RMSE diff (SRH - MR_ENS)

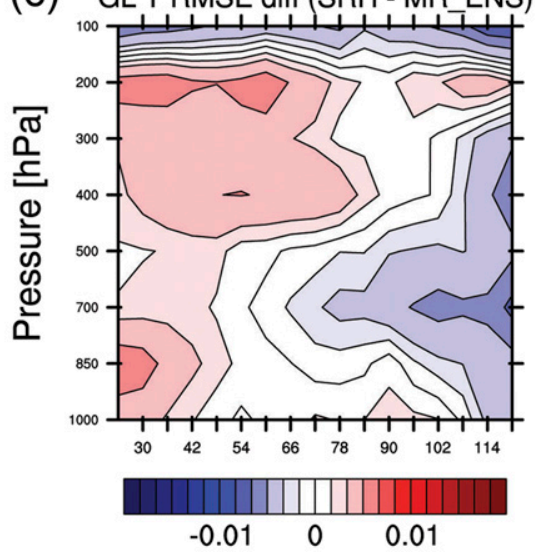

Fcst hours [h]
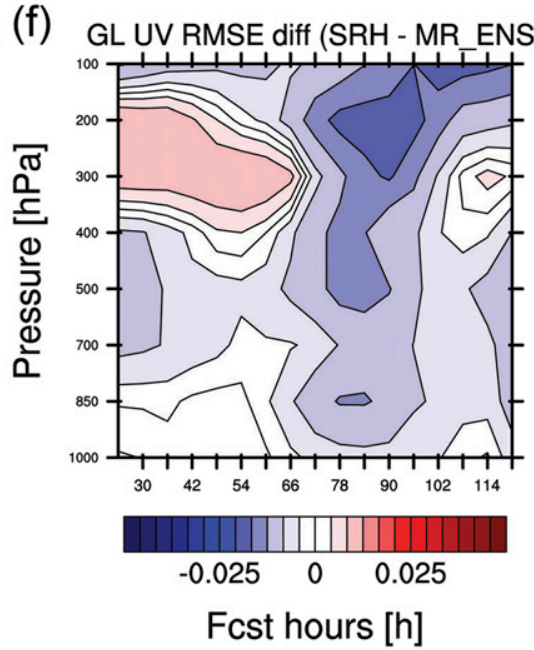

FIG. 4. The differences of the globally averaged RMSEs of 1-5 day forecasts verified against the ECMWF interim reanalysis between (left) SR-Low and MR-ENS, (middle) DR and MR-ENS, and (right) SR-High and MR-ENS for (a)-(c) temperature and (d)-(f) wind as a function of pressure level and forecast lead time. The black dots indicate the level where MR-ENS statistically significantly differ from the other experiments at or above the $95 \%$ confidence level by applying the paired $t$ test.

impact of the resolution of BECs is further compared through verifying the TC track forecasts. A total of 12 TCs were observed during the experimental period. The NCEP tropical cyclone tracker (Marchok 2002) was used to track the tropical cyclone forecasts. Figure 5 shows the TC track forecast error averaged over all cases. DR outperforms SR-Low at longer lead times. Given the methodological difference between DR and SR-Low, this result suggests the environmental field could be more accurately analyzed by the high-resolution background and the high-resolution static BEC, even though there is no contribution from the flow-dependent ensemble covariance at small scales. MR-ENS outperforms DR with statistical significance up to the $60 \mathrm{~h}$ lead times. Although DR outperforms MR-ENS after the 90-h lead time, their difference is statistically insignificant. Given the methodological difference between DR and MR-ENS, this result suggests that the use of the high-resolution flow-dependent BEC further improves the analyzed environment of the TC which improves the subsequent TC track forecasts. Note that unlike the global forecast evaluation, for the TC track forecast, the improvement of MR-ENS relative to SR-Low are attributed not only to the high-resolution background ensemble but also the high-resolution background and static covariance. Consistent with the global forecast evaluation, MR-ENS shows similar TC track forecast errors as SR-High though MR-ENS is computationally less expensive.

\section{d. Evaluation of the background ensemble spread}

In the ensemble-based data assimilation, the background ensemble spread together with the estimated 


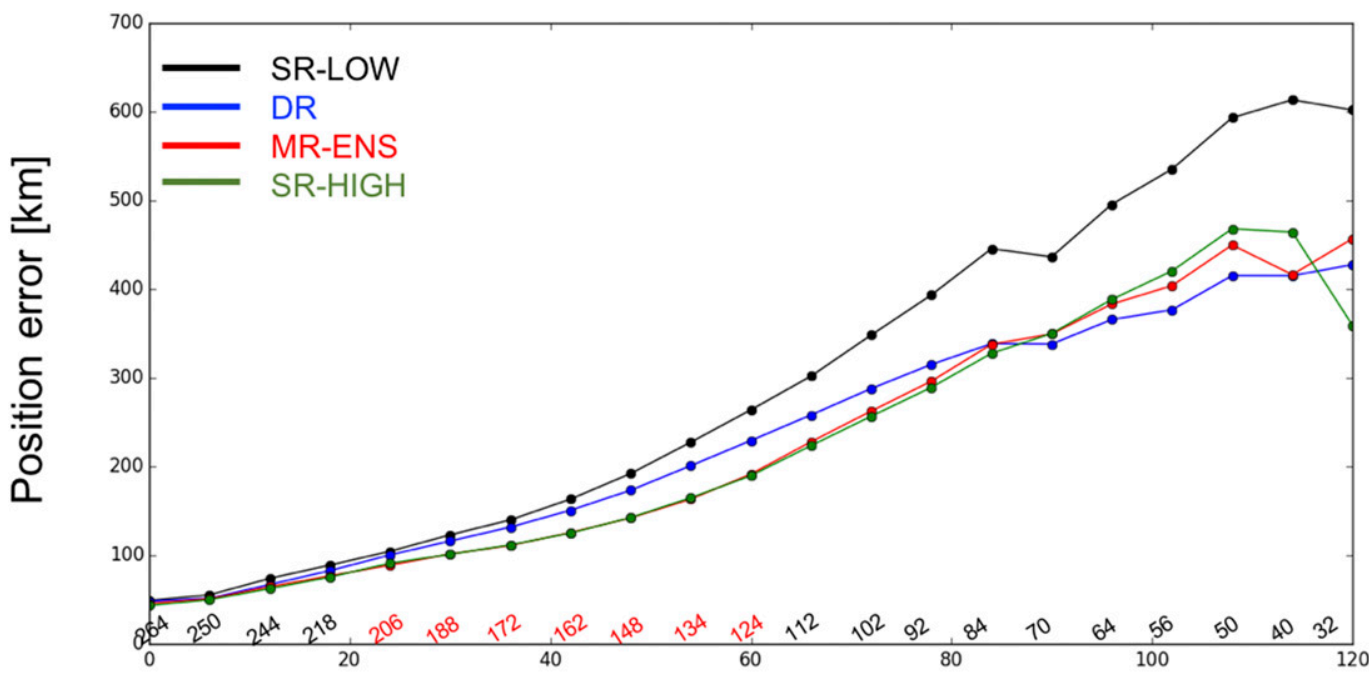

Forecast time $[\mathrm{h}]$

FIG. 5. The RMSEs of tropical cyclone track forecasts for SR-Low (black dotted line), DR (blue dotted line), MR-ENS (red dotted line), and SR-High (green dotted line). The number above the $x$ axis denotes the total number of sample sizes used in the calculation at each forecast lead time over the experimental period and red color denotes the lead times with statistical significance at or above the $95 \%$ confidence level from the paired $t$ test between MR-ENS and DR.

observation errors determines how much penalty will be imposed on the model background. Figure 6 shows the 6-h background ensemble spread and the corresponding RMS fit of the background ensemble mean to the rawinsonde observations as a function of pressure levels. Overall the spread and background error relationship shown in Fig. 6 is consistent with early studies (e.g., Wang et al. 2013 and Huang and Wang 2018).
The ensemble spread for temperature is overall underdispersive especially in the lower and upper troposphere. The wind ensemble spread is overdispersive in the middle of the troposphere and underdispersive in the lower and upper troposphere. For temperature, the RMS fit to rawinsonde and the background ensemble spread are nearly indistinguishable for the four experiments. For wind, SR-High shows the smallest RMSE followed by

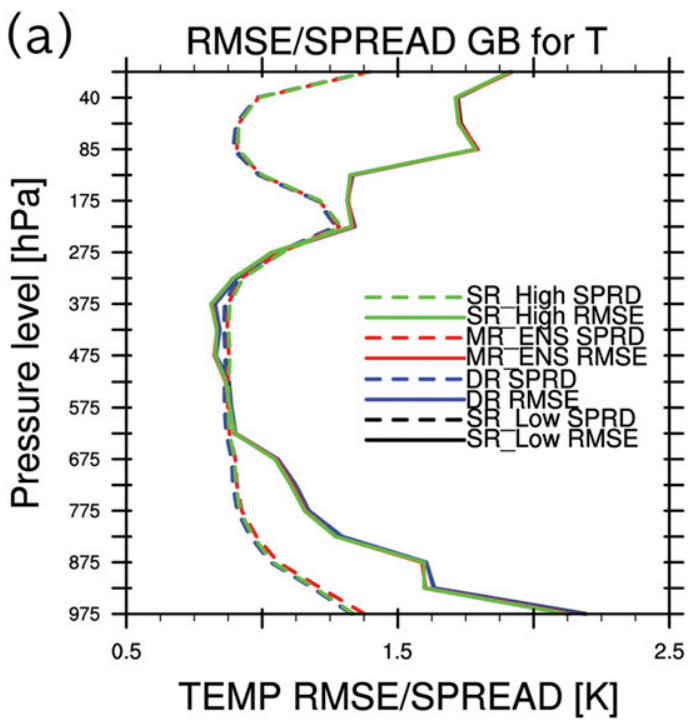

(b)

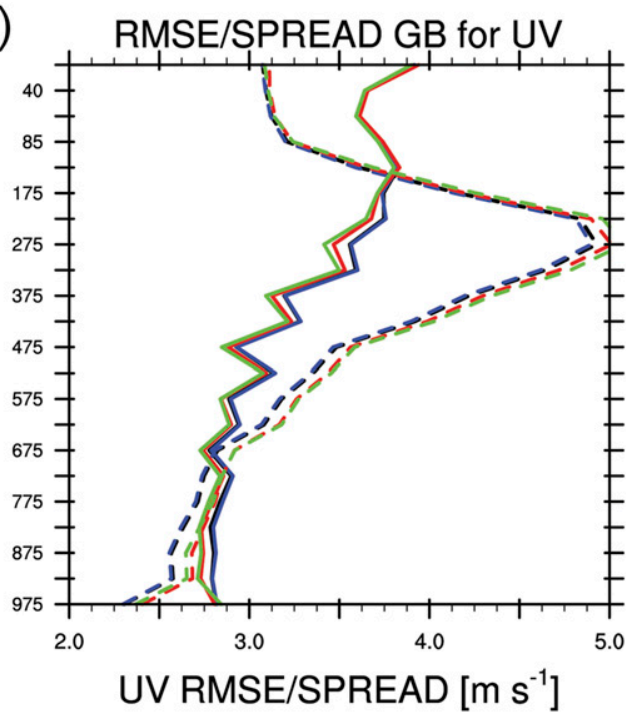

FIG. 6. Vertical profiles of the square root of the globally and temporally averaged innovation variance (solid lines) and the square root of the observation error variance plus the 6-h background ensemble variance (dashed lines) in SR-Low (black), DR (blue), MR-ENS (red), and SR-High (green). 
MR-ENS. Consistently, the ensemble spread from SRHigh is slightly larger than MR-ENS. SR-Low and DR are nearly indistinguishable for both the RMSE and the ensemble spread. They both have larger RMSE and smaller ensemble spread than SR-High and MRENS. This result indicates that the increase of ensemble resolution is beneficial for enhancing the ensemble spread especially for the wind field. As shown later in section 4f, the larger total ensemble spread in MR-ENS and SR-High is primarily because the adopted highresolution background ensemble has larger variance over small scales that are unresolved by the lowerresolution ensemble. As also shown in sections $4 \mathrm{~g}$ and $4 \mathrm{~h}$, using high-resolution background ensemble increases the rank and improves the background error correlation estimation. These aforementioned benefits cannot be achieved by simply applying an inflation factor to the coarse resolution ensemble.

\section{e. Impact of the high-resolution ensemble on variational minimization}

Figure 7 shows the evolution of the observation term of the cost function ( $\mathrm{Jo}$ ) during the minimization for the first and second outer loops averaged over the experimental period. This term is defined as the fourth term on the right-hand side in Eq. (5). The Jo term indicates the fit of a background state to the observation and decreases rapidly during the minimization iteration as the background state approaches to the observation. In Fig. 7, 50 and 150 iterations are shown for the first and second outer loop, respectively. The Jo term at the last iteration in the second outer loop is considered as the analysis fit to observations.

In general, the degree to which the analysis fits to the observation is determined by the accuracy of the background forecast and the relative magnitude of the background error variance and the observation error variance (Wang et al. 2013). SR-High shows the smallest Jo at the final iteration. This result is consistent with the fact that the background of SR-High is the most accurate (Fig. 3) and the ensemble spread of the SR-High is the largest (Fig. 6). These results can be attributed to SR-High being able to estimate the ensemble BEC on small scales. MR-ENS shows larger Jo than SR-High, but smaller Jo than DR and SR-Low in both the first and the second outer loops. This result is also consistent with that MR-ENS shows larger ensemble spread and more accurate background than SR-Low and DR (Fig. 6). DR shows smaller Jo and closer fit of the analysis to the observations than SR-Low (Fig. 7), which can be attributed to the use of the high-resolution (T670) control background and the high-resolution static covariance in DR than SR-Low.

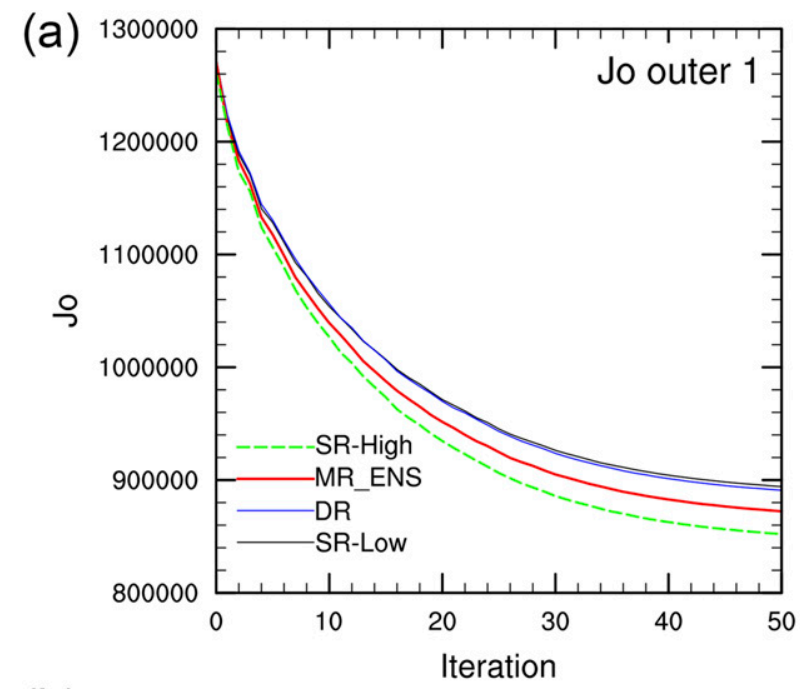

(b)

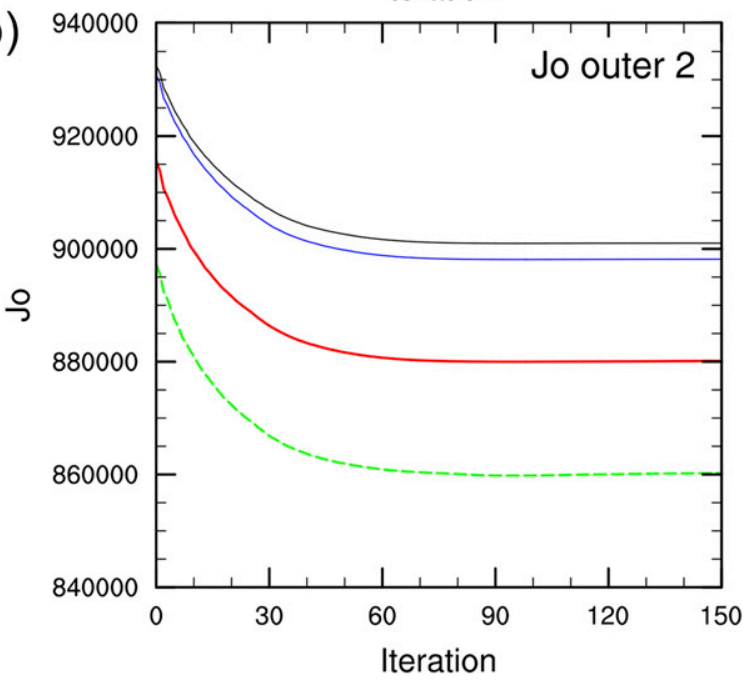

FIG. 7. The values of the observation term of the cost function (Jo) as a function of the minimization iteration for SR-Low (solid black), DR (solid blue), MR-ENS (solid red), and SR-High (solid green) in the (a) first outer loop and (b) second outer loop.

\section{f. Scale-dependent evaluation of the analysis increment}

To evaluate the impact of the resolution of the BECs on the analysis increment, both the kinetic energy power spectra and the wavelet diagnostics are performed. Early studies used similar methods to diagnose either ensemble-based data assimilation or ensemble perturbation design (e.g., Zhang et al. 2006; Meng and Zhang 2007; Johnson et al. 2014). Figure 8 shows the kinetic energy power spectra for the analysis increment averaged over the globe during the experimental period. The power of the analysis increment from all experiments show similar magnitude for wavenumbers less than about 100. As expected, the experiments using the high-resolution ensembles (MR-ENS and SR-High) and 

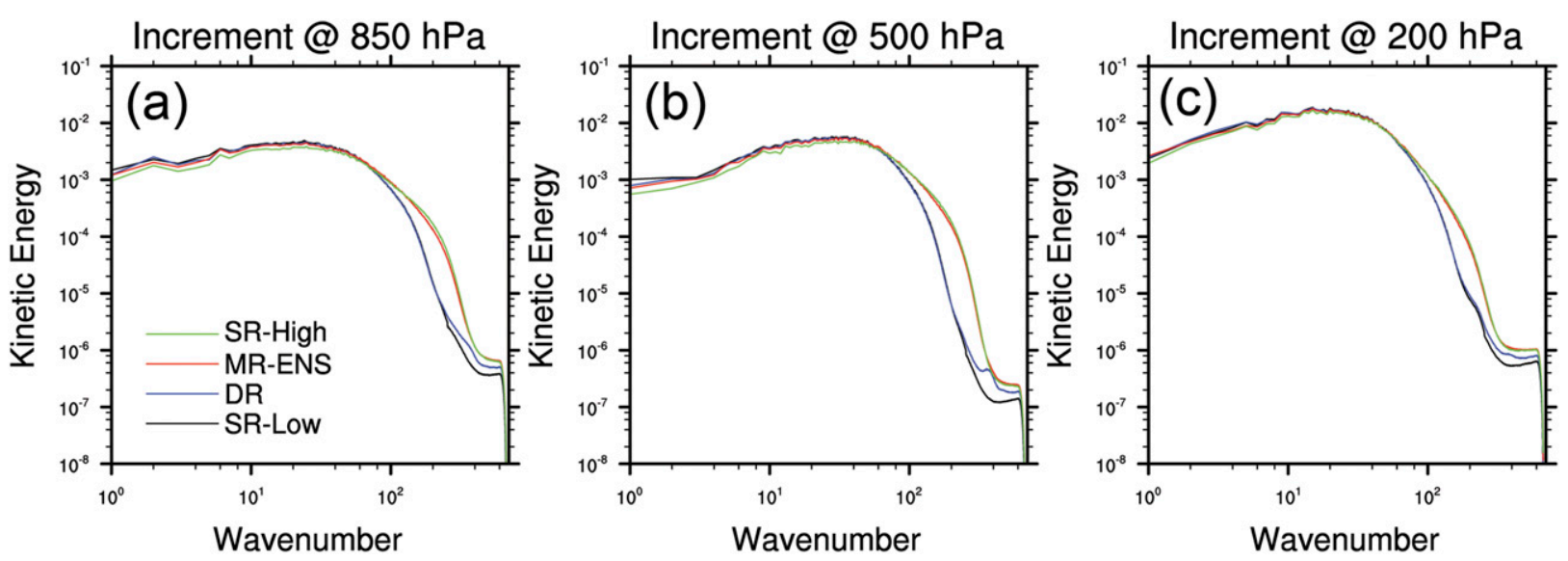

FIG. 8. The power spectra of kinetic energy for analysis increment $\left(\mathrm{m}^{2} \mathrm{~s}^{-2}\right)$ for SR-Low (solid black), DR (solid blue), MR-ENS (solid red), and SR-High (solid green) at (a) 850, (b) 500, and (c) $200 \mathrm{hPa}$.

the low-resolution ensembles (SR-Low and DR) show a pronounced difference for wavenumbers greater than 100 , due to the lack of contribution to the analysis increment from the ensemble BEC on small scales in both SR-Low and DR. Note that the difference starts to show at wavenumber 100 , which is smaller than the truncation wavenumber, 254, of the low-resolution ensemble. This is because the effective spatial resolution of the ensemble, at which the forecast error covariance is estimated, is lower than the nominal resolution T254 (Bonavita et al. 2011). The SR-High shows slightly larger increment than MR-ENS for wavenumbers greater than 100. The difference between MR-ENS and SR-High, however, is much smaller compared to the difference between the experiments using high- and low-resolution ensembles. This suggests that although MR-ENS uses less number of high-resolution ensemble members compared to SRHigh, the reduced number of high-resolution ensemble members in MR-ENS introduces a quantitatively similar contribution to the analysis increment compared to SRHigh at small scales. The analysis increment of SR-Low is smaller than DR for wavenumbers greater than about 200 because there is no contribution to the analysis increment from both the static and ensemble BECs on small scales in SR-Low. It is also noted that DR shows the sharp decline and rise of analysis increment for wavenumbers greater than the truncation wavenumber, 254 , of the low-resolution ensemble, which is likely due to the accumulation of aliasing errors caused by the interpolation of low-resolution ensembles to high resolution at every iteration (Kleist 2012).

The impact of the resolution of the BEC on the analysis increment can be dependent on the nature of the dynamic and physical processes of the region. For example, relatively smaller scale convective instabilities may dominate the forecast error in the tropics than midlatitudes
(Reichler and Roads 2005; Straus and Paolino 2008). Therefore, wavelet analysis was applied on the analysis increment to analyze variations of power as a function of location and scale (Torrence and Compo 1998). The kinetic energy of the analysis increment at a latitude is decomposed into the longitude-wavenumber space using Morlet wavelets (Domingues et al. 2005), and then are averaged over the Northern Hemisphere $\left(\mathrm{NH} ; 20^{\circ}-90^{\circ} \mathrm{N}\right)$, the tropical region (TR; $\left.20^{\circ} \mathrm{S}-20^{\circ} \mathrm{N}\right)$, and Southern Hemisphere $\left(\mathrm{SH} ; 20^{\circ}-90^{\circ} \mathrm{S}\right)$. The comparison of the analysis increments among $\mathrm{NH}$ and $\mathrm{SH}$ reveals much smaller overall differences among the four experiments for all scales considered (not shown) compared to the tropical region, although at scales smaller than the wavenumber of 100, SR-High and MRENS show more apparently larger increments than DR and SR-Low. Therefore this section focuses on the comparison of the increment over TR (Fig. 9). Red and blue indicate high- and low-wavelet power spectra, respectively. The scale ranges from $2^{\circ}$ to $2^{(n / 4)}$, where $n$ is the total number of scales. In this study, we choose a total of 40 scales so the increment is decomposed into scales ranging from 1 to 1024 , which corresponds to about 8000 to $25 \mathrm{~km}$ in wavelength. Analogous to the more familiar Fourier decomposition, we add the wavelength on the right side of the $y$ axis as well as the wavenumber on the left side of the $y$ axis.

In Figs. 9a-d, the increments from all four experiments in general show two peaks at longitude $90^{\circ}-120^{\circ} \mathrm{E}$ and around longitude $60^{\circ} \mathrm{W}$ for smaller scales (wavenumber greater than 10). This pattern is consistent with the two convection peaks shown in monthly mean outgoing longwave radiation (OLR) provided from the NOAA (https:// www.ncdc.noaa.gov/cdr/atmospheric/outgoing-longwaveradiation-monthly; http://apdrc.soest.hawaii.edu/datadoc/ noaaolr_month.php) (Fig. 9e). Monthly mean OLR (Liebmann and Smith 1996) indicates that there were 
(a) SR-Low

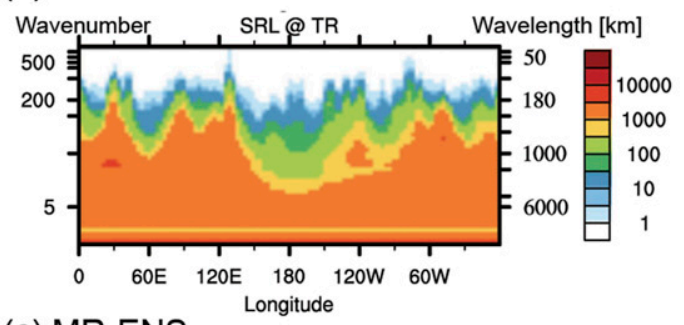

(c) MR-ENS

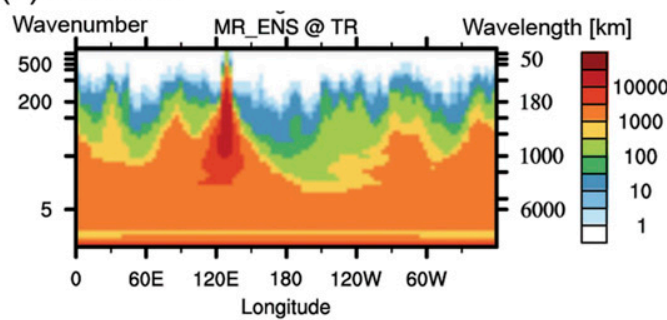

(e) Monthly mean OLR [Wm-2]

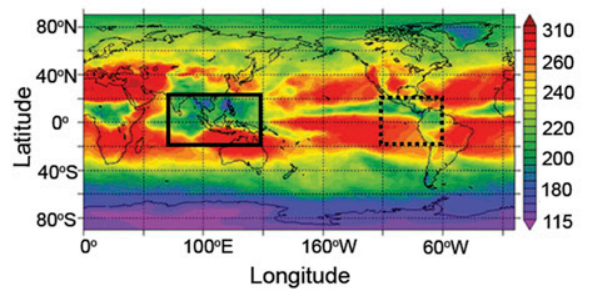

(b) DR

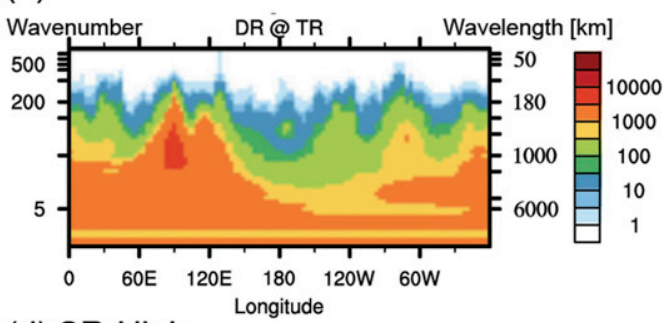

(d) SR-High

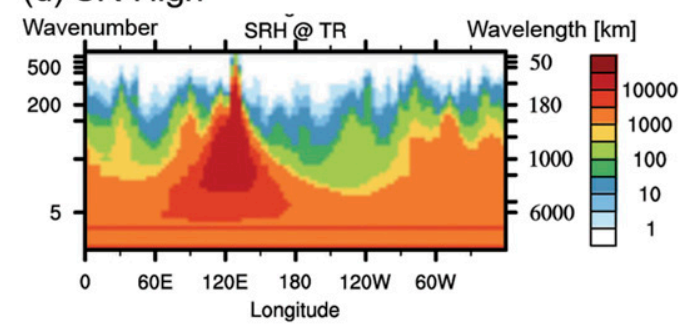

FIG. 9. Wavelet analysis of kinetic energy analysis increment for (a) SR-Low, (b) DR, (c) MR-ENS, and (d) SR-High averaged over the tropical region (TR). The $x$ axis represents longitude and the left $y$ axis represents wavenumber and the right $y$ axis the corresponding wavelength $(\mathrm{km})$. (e) Monthly mean OLR $\left(\mathrm{W} \mathrm{m}^{-2}\right)$ with $2.5^{\circ}$ resolution over the experimental period. Black solid and dotted box represent the TC active-area over the tropical western Pacific Ocean and over the tropical eastern Atlantic Ocean, respectively.

strong convections associated with six tropical cyclones (Mangkut, Utor, Trami, Pewa, Unala, Kong-rey) over the west Pacific Ocean between $60^{\circ}$ and $150^{\circ} \mathrm{E}$ (surrounded by black solid box) during the experiment period (Fig. 9e). During the same period, two TCs at a weaker, tropical storm category occurred in the Atlantic Ocean (Erin and Fernand) (surrounded by black dotted box) (Fig. 9e). Therefore, the monthly mean OLR shows two peaks of convection at the tropical western Pacific Ocean and the tropical eastern Atlantic Ocean, with the Atlantic peak weaker than the Pacific peak.

The SR-High increment shows prominently larger power within longitude $90^{\circ}-150^{\circ} \mathrm{E}$ at almost all scales compared to the other three experiments. This is consistent with the stronger convective activities in reality demonstrated in monthly mean OLR this region. MR-ENS shows relatively weaker power than the SR-high, but still shows much higher increment than SR-Low and DR in this region. DR has a slightly larger power within 100-200 wavenumbers near longitude $90^{\circ} \mathrm{E}$ than SR-Low, which could be attributed by the high-resolution static BEC.

These results imply that when underlying dynamic or physical processes possess small scales, the high-resolution flow-dependent ensemble covariance is beneficial to reflect the small-scale background errors. MR-ENS even having partial ensemble member at high resolution can alleviate the underrepresentation of the small-scale errors by DR and SR-Low while saving the computing cost compared to SR-High.

\section{g. Ensemble dimension-Effective rank of ensemble covariance}

Given a limited number of ensemble members, it is desirable that each member is an independent sample to the background error (Wang and Bishop 2003). In this study, all experiments use the same background ensemble size of 80 . In this section, we evaluate how the resolution of the ensemble impacts the effective rank of the background ensemble covariance by calculating the Ensemble dimension (E-dimension) (Patil et al. 2001; 

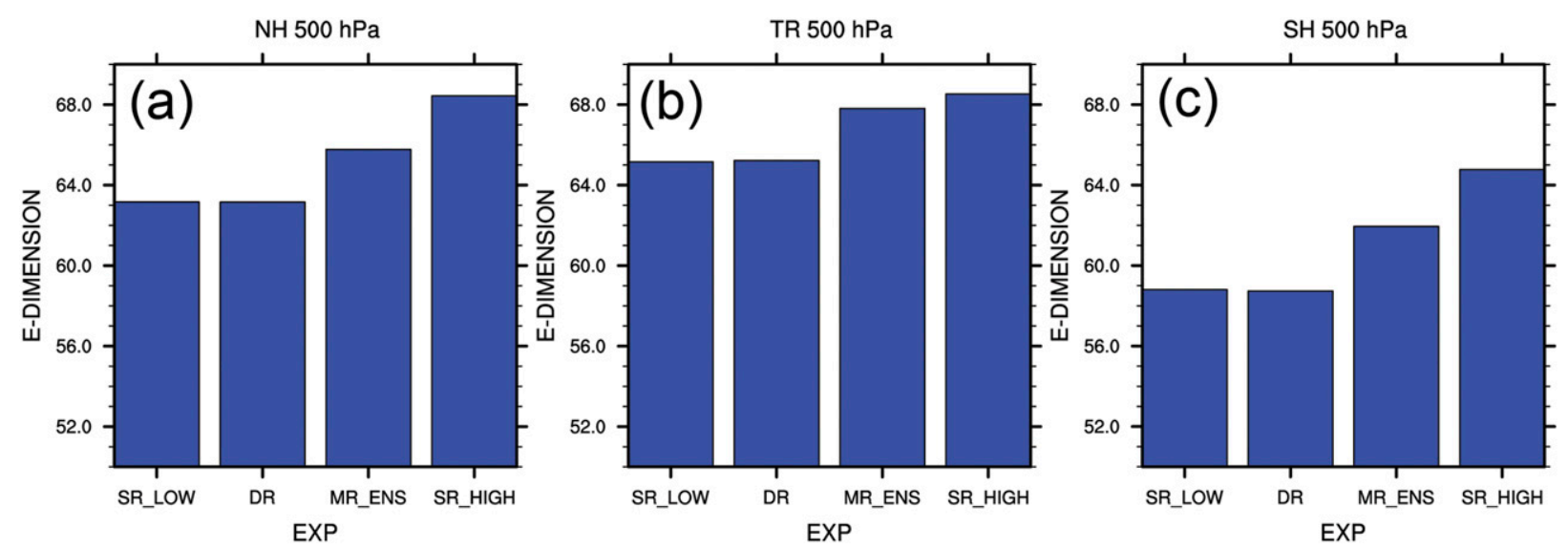

FIG. 10. Ensemble E-dimension in (a) NH, (b) TR, and (c) SH at $500 \mathrm{hPa}$ averaged for the experiment period.

Kay and Kim 2014; Huang and Wang 2018). Figure 10 shows the averaged E-dimension corresponding to the 6-h background ensemble covariances of zonal wind and temperature for each hemisphere at $500 \mathrm{hPa}$ during the experimental period.

All of the experiments generally have the smallest E-dimension in SH than in NH and TR (Fig. 10). The winter $\mathrm{SH}$ is usually dominated by high baroclinic instability, which would lead to faster error growth (Straus and Paolino 2008; Liu et al. 2009). Previous studies (Kuhl et al. 2007; Kay and Kim 2014) have found the larger error growth is often accompanied with smaller E-dimension.

In all regions, SR-High has the largest E-dimension followed by MR-ENS. SR-Low and DR have similarly lower E-dimension (Fig. 10). This result is consistent with the expectation that using the high-resolution ensemble increases the opportunity to sample errors with a wider range of scales and therefore better samples independent errors. If we further evaluate the regional characteristics of the E-dimension, it is notable that the difference of the E-dimension between MRENS and SR-High is smaller in the TR region than in the $\mathrm{NH}$ and $\mathrm{SH}$ regions (Fig. 10b). This result together with the wavelet diagnostics (Fig. 9) implies that increasing the resolution for part of the ensemble members in the 4DEnVar is likely more effective to sample the small-scale errors independently in the $\mathrm{TR}$ region than in the other regions. The $\mathrm{E}$ dimension of the 40 low-resolution ensemble members and the 40 high-resolution ensemble members of MR-ENS are compared with each other. Although they have the same number of members, the higher resolution component has an $\mathrm{E}$ dimension of 36.2 whereas the low-resolution component has an $\mathrm{E}$ dimension of 34.1. These results confirm that the use of partial high-resolution ensemble member contributes to the higher rank in MR-ENS than DR/SR-Low.

\section{h. Quantitative evaluation of the accuracy of the background ensemble correlation}

As the resolution of the background ensemble decreases, all the fine structure of the ensemble correlation associated with the small scales is neglected, which offsets the information contained in the high-resolution observation in the DA process. Therefore, in this section, we evaluate the impact of ensemble resolution on the accuracy of the background ensemble correlation estimation.

Generally, the high-resolution background ensemble correlation (MR-ENS, SR-High) shows a multiscale structure whereas the low-resolution background ensemble correlation (SR-Low, DR) shows a large-scale smoothed structure. An example associated with a front is discussed below. The auto- correlation of the zonal wind at the point marked by the black star with the zonal wind at other regions are shown in Fig. 11. In the midlatitudes and polar regions where the dynamics is largely determined by synoptic-scale baroclinic instabilities (Holton 2004), the low-resolution ensemble is able to represent the large-scale correlation of the system. However, for example, in the subsynoptic frontal region where a diabatic process of a smaller scale is active along the front, only the high-resolution ensemble is able to represent the multiscale correlation associated with the fronts. SR-Low and the DR show a relatively large-scale autocorrelation that is uniform and smoothed in the east-west direction, parallel to the frontal zone (Figs. 11a,b). MR-ENS and SR-High not only show the same large-scale correlation elongated in the east-west direction along the front, they both also show an oscillation between positive and negative correlation values across the front (Figs. 11c,d). These smaller scale correlation structures of MR-ENS 
(a) SR-Low 850 hPa UU correlation

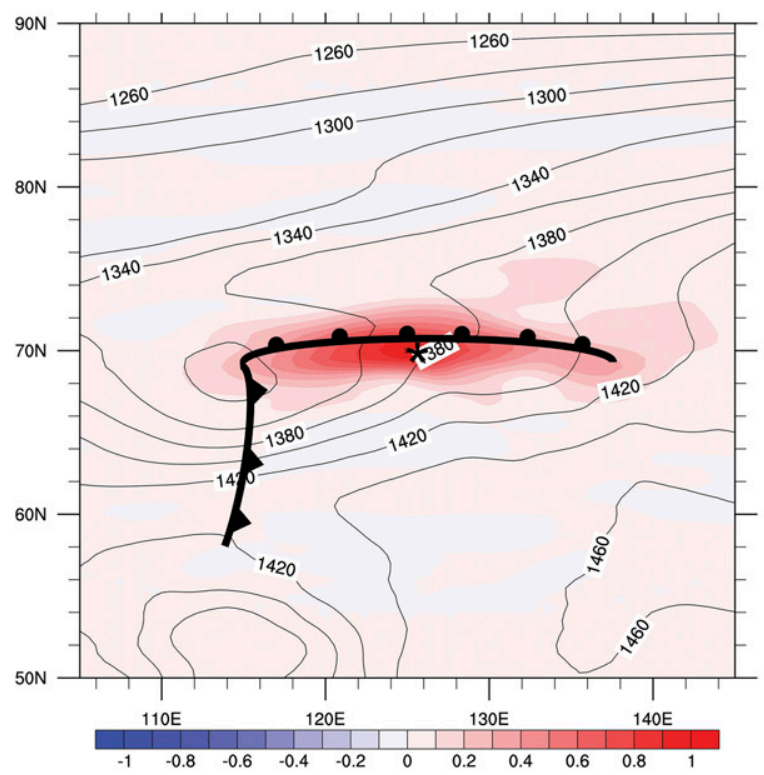

(c)

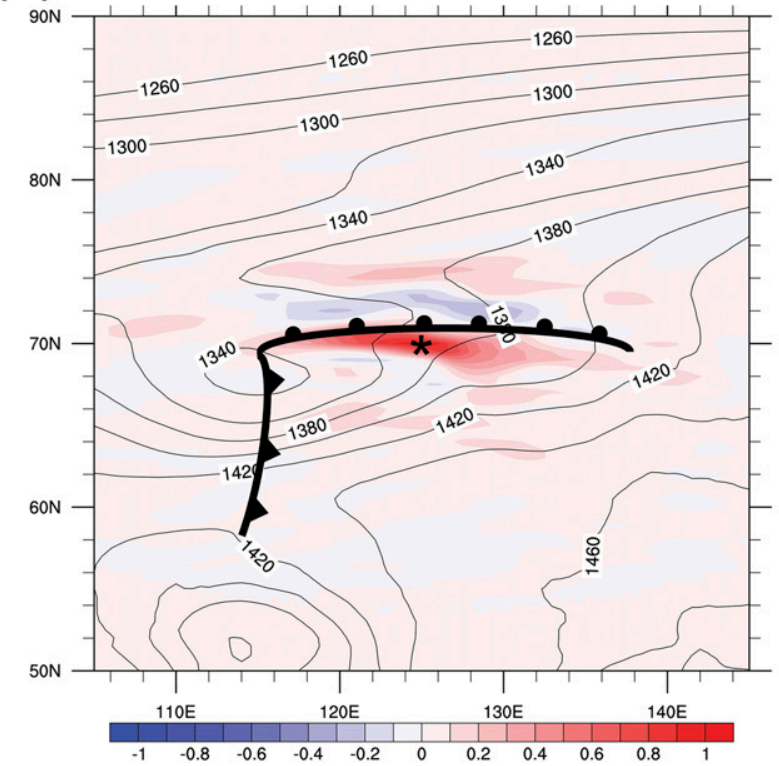

(b) DR $850 \mathrm{hPa}$ UU correlation

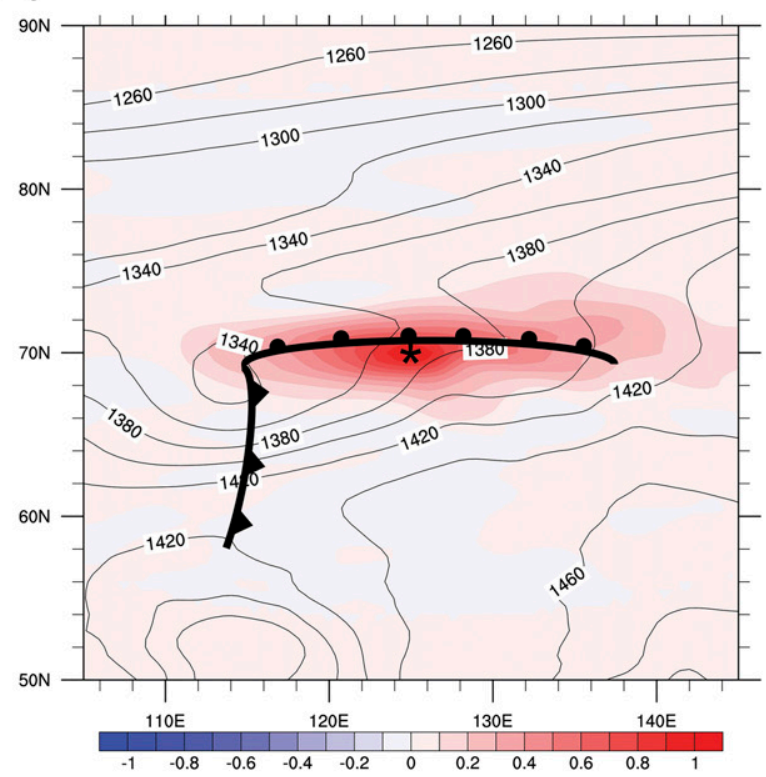

(d)
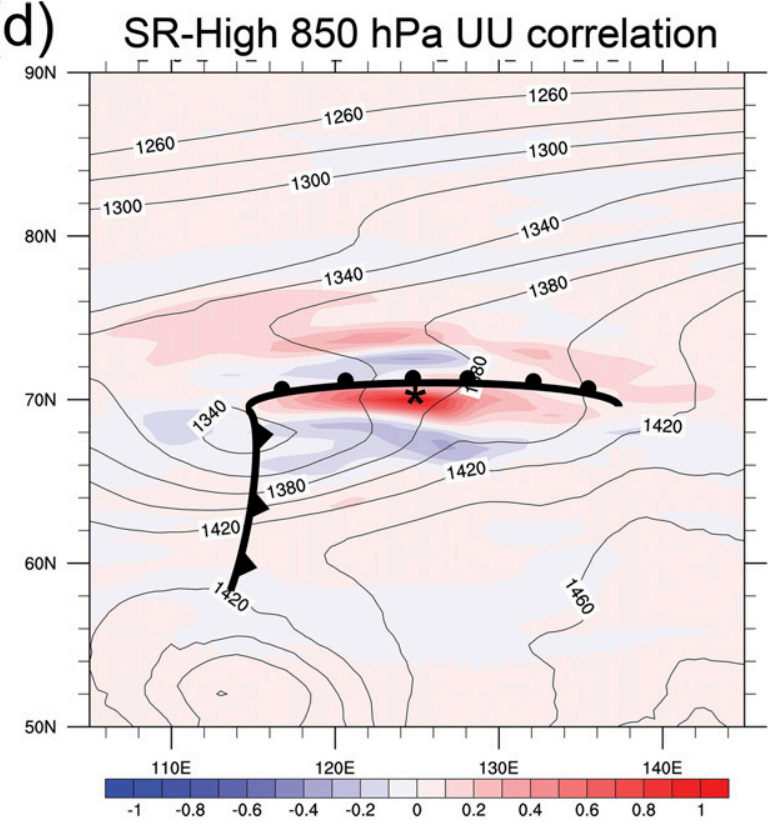

FIG. 11. The 850 -hPa zonal wind ensemble autocorrelations (color shaded) between the central grid point (marked by the black star) and other grid points calculated from the original 6-h background ensemble in (a) SR-Low, (b) DR, (c) MR-ENS, and the (d) SR-High. The solid black contours represent the geopotential heights of the 6-h background ensemble mean at $850 \mathrm{hPa}$. The surface frontal position is marked with solid black lines.

and SR-High reflects the variation of the wind across the front. Specifically, the correlation suggests the correction of the wind shear associated with the warm front if observations located at the star were assimilated.

To systematically evaluate the effect of the ensemble resolution on the correlation accuracy, we define a new score which aims to evaluate the accuracy of the ensemble correlation as a function of distance. We denote the score as the absolute value of the correlation error (ACE):

$$
\mathrm{ACE}(\mathrm{EXP})=\operatorname{abs}[\operatorname{Corr}(\mathrm{REF})-\operatorname{Corr}(\mathrm{EXP})] \text {, }
$$

where abs is a function to return the absolute value, Corr represents the correlation estimated by using the 

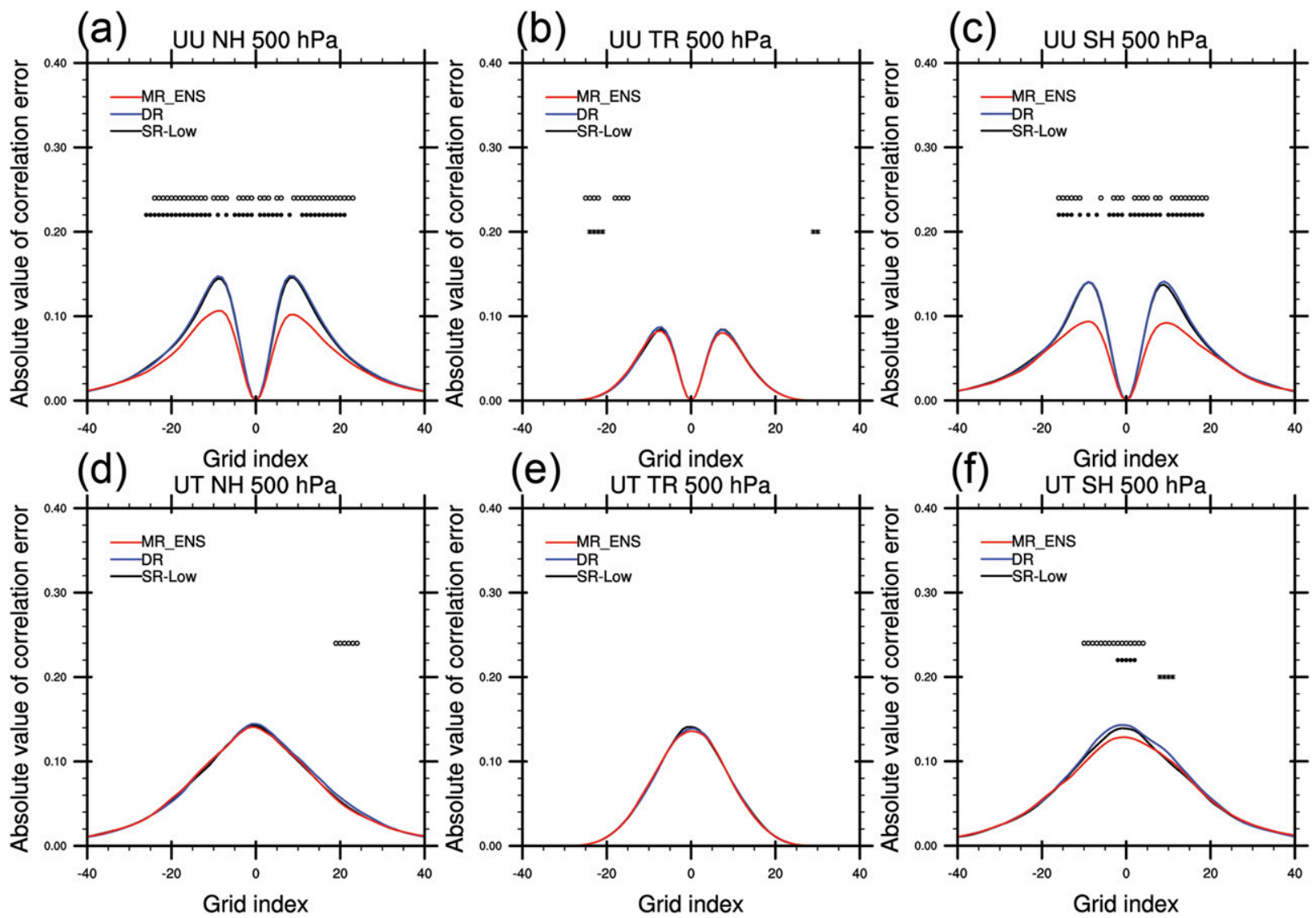

(e) Grid index
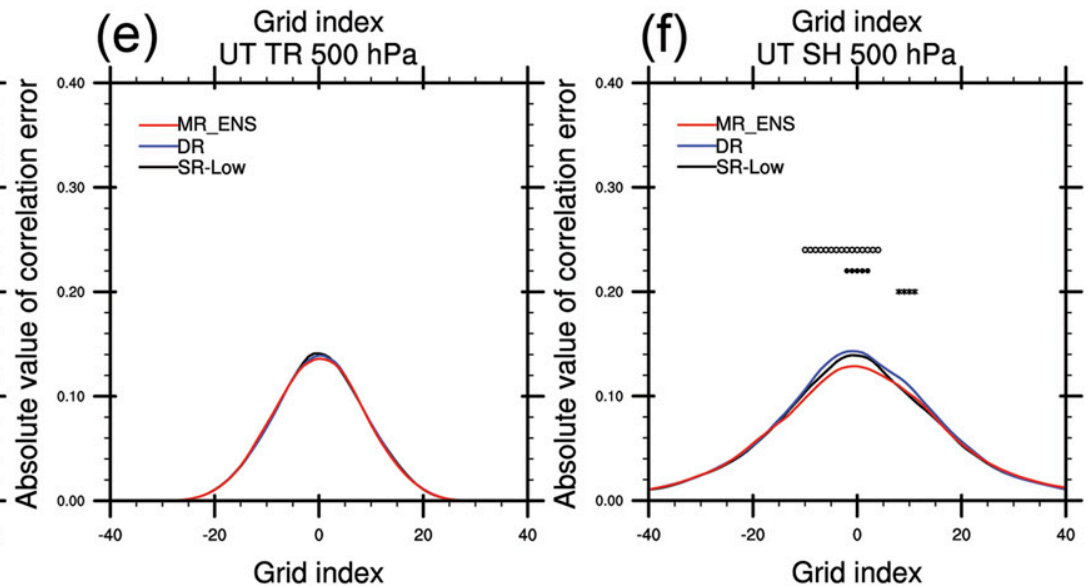

FIG. 12. Globally averaged ACEs from SR-Low (solid black), DR (solid blue), and MR-ENS (solid red) calculated from the large-scale 6-h background ensemble perturbations for the zonal wind autocorrelation at $500 \mathrm{hPa}$ averaged over (a) NH, (b) TR, and (c) SH for the temperature autocorrelation averaged for the experiment period. The asterisk, filled circle, and open circle indicate that the ACE differences between SR-Low and DR, SR-Low and MR-ENS, and DR and MR-ENS are statistically significant at or above the 95\% confidence level by applying the paired $t$ test at the corresponding grid index. (d)-(f) As in (a)-(c), but for the cross correlation between temperature and zonal wind.

background ensemble, REF refers to SR-High, and EXP denotes the experiments of SR-Low, DR, and MR-ENS. In this score, the SR-High experiment is used as a referencing truth because it shows the most accurate forecast in terms of the fit of the background to observations, longer term global forecast and TC track forecast (Figs. 3-5). The accuracy of the ensemble correlation as a function of the scales is further evaluated as followed. Using Fourier filtering (Bloomfield 2002), the background ensemble is decomposed into two scales, $\mathbf{X}=$ $\mathbf{X}_{L}+\mathbf{X}_{S}$ where $\mathbf{X}$ is a 6-h background ensemble, $\mathbf{X}_{L}$ represents a large-scale background ensemble whose wavenumber ranges from 1 to $80 . \mathbf{X}_{L}$ is considered as synoptic-scale background ranging in scales from about 2000 to $500 \mathrm{~km}$. $\mathbf{X}_{S}$ is the remaining small-scale background ensemble whose wavenumber is large than 80 , and is representing mesoscales ranging from about 500 to $40 \mathrm{~km}$ (Caron and Buehner 2018). The large- and smallscale ensemble correlations are then calculated over an area of $4000 \mathrm{~km}$ by $4000 \mathrm{~km}$ where the size of $4000 \mathrm{~km}$ is approximately equal to the localization cutoff radius. Given the ensemble BECs are modulated by the localization function during the variational update, we evaluate the accuracy of the ensemble correlation with the covariance localization applied. For each of the NH, TR, and $\mathrm{SH}$, the ACEs are calculated by averaging the samples collected at 55 evenly spaced areas for each 6-h background ensemble.

Figure 12 shows the ACEs for the ensemble autocorrelation of the zonal wind, and cross-correlation between the zonal wind and the temperature as a function of distance for large scales. The autocorrelation errors of all experiments are zero at the center grid point because they all have a correlation of 1.0. At the longer distance, the ACEs taper to zero due to the application of the covariance localization function. MR-ENS shows smaller autocorrelation error than SR-Low and DR in both $\mathrm{NH}$ and $\mathrm{SH}$. The improvement peaks near the 10 th grid 

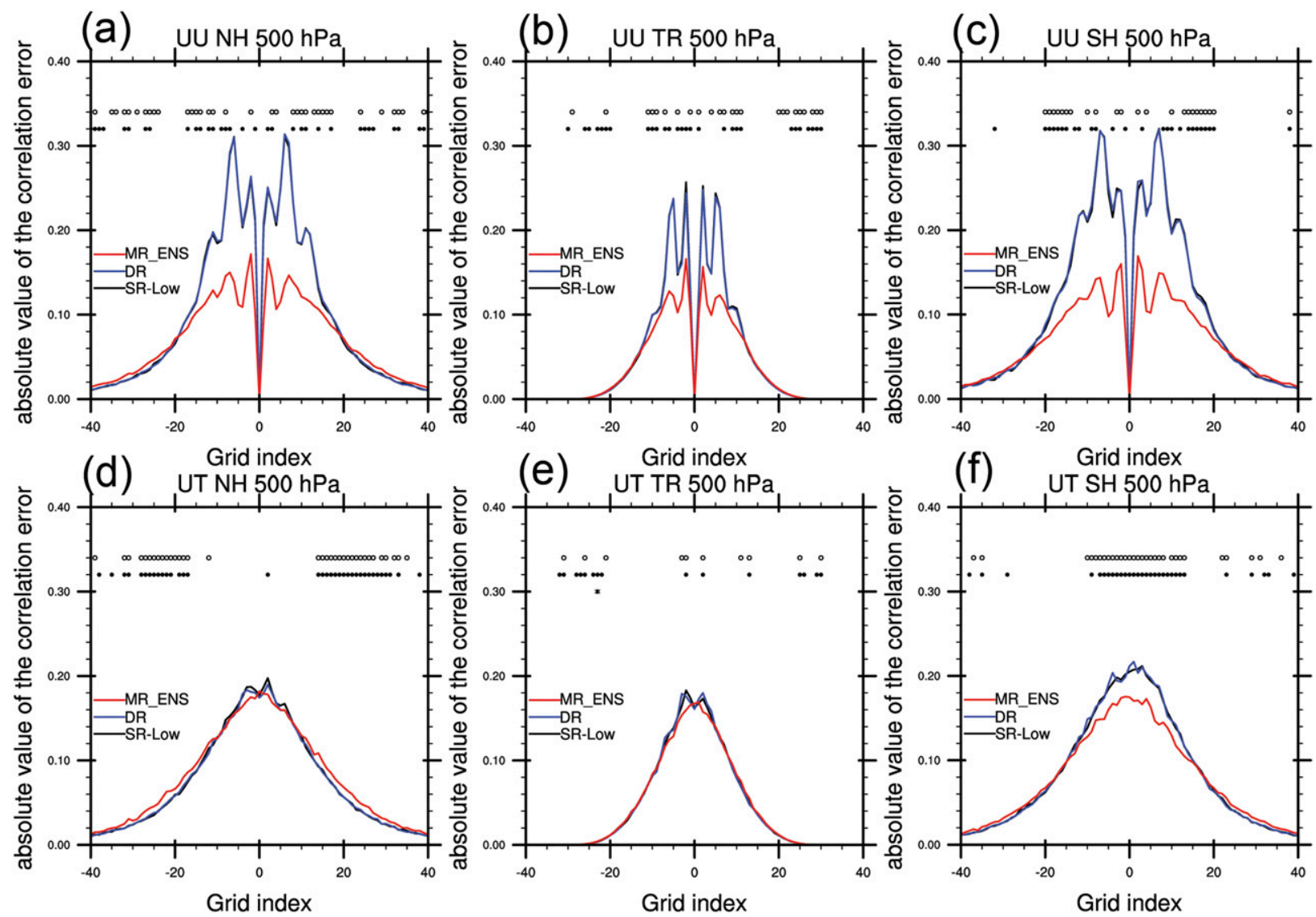

FIG. 13. As in Fig. 12, but for ACEs from the small-scale 6-h background ensemble perturbations.

index (about $500 \mathrm{~km}$ from the center point). MR-ENS, SR-Low, and DR show a comparable accuracy of autocorrelation in TR. In contrast to the autocorrelation, the error of the estimated cross-correlation peaks at the central grid point. The errors of cross-correlation are nearly indistinguishable for the three experiments in NH and TR. MR-ENS shows a slight improvement in SH. Figure 13 shows the ACEs of the auto- and crosscorrelation derived from the small-scale ensemble. Comparing Figs. 12 and 13 shows that the small-scale ensemble inherits a larger correlation error than the large-scale ensemble. Different from large scales, MRENS improves the autocorrelation compared to DR and SR-Low not only in NH and SH but also in TR. Similar to large scales, all three experiments are nearly indistinguishable for cross-correlation except MR-ENS shows a slight improvement in SH. Compared to the autocorrelation, MR-ENS shows less improvement on the crosscorrelations for both the large- and small-scales. This is likely because the ensemble-estimated cross-correlation is more prone to the sampling error than autocorrelation (Huang and Wang 2018). Meanwhile, MR-ENS shows larger relative improvement in the cross-correlation over the other experiments at small scale than at large-scale (Figs. 12 and 13). This is attributed to the high-resolution background ensemble used by MR-ENS. In summary, MR-ENS improves the autocorrelation derived from both the small (meso)- and large (synoptic)-scale ensembles in both the $\mathrm{NH}$ and $\mathrm{SH}$ relative to SR-Low and DR. In the TR region, increasing the resolution of part of the background ensemble members is particularly effective to reduce the large error of the autocorrelation derived from the small-scale ensemble. Because SR-Low and DR use the same resolution for the background ensemble, they have similar auto- and crosscorrelation errors regardless of regions and scales.

\section{i. Comparison of computational costs}

In this section, we compare the computational costs of MR-ENS with other methods in a single 4DEnVar cycle. The computational cost is derived by multiplying the wall clock time and the number of cores used while the experiments are performed on the National Oceanic and Atmospheric Administration (NOAA) High-Performance Computing System Jet. The control forecast is ignored in estimating the computational cost because its costs is 
TABLE 2. Computational cost derived by multiplying wall clock time ( $\mathrm{min}$ ) and the number of cores used in each component of a single 4DEnVar DA cycle. Costs of all experiments were estimated based on xJet node on the NOAA High-Performance Computing System Jet machine. Cost is estimated with I/O ignored.

\begin{tabular}{|c|c|c|c|c|c|}
\hline \multirow[b]{2}{*}{ Experiment } & \multicolumn{3}{|c|}{ Cost in a single 4 DEnVar cycle [Wall clock time $(\mathrm{min}) \times$ cores] } & \multirow[b]{2}{*}{ Total cost } & \multirow[b]{2}{*}{ Total cost ratio relative to SR-Low } \\
\hline & 4DEnVar update & EnKF update & Background ensemble & & \\
\hline SR-Low & 12000 & 16800 & 16800 & 45600 & 1 \\
\hline DR & 21600 & 16800 & 16800 & 55200 & 1.21 \\
\hline MR-ENS & 46560 & 16800 & 65184 & 128544 & 2.81 \\
\hline SR-High & 81120 & 16800 & 113568 & 211488 & 4.63 \\
\hline
\end{tabular}

negligible compared to the total cost of a cycle. Because the cost of I/O highly depends on the frequency of output, the choice of output variable, and I/O techniques such as parallel I/O (Balle and Johnsen 2016), we do not include the $\mathrm{I} / \mathrm{O}$ cost in the estimate. It is worth noting that following Lei and Whitaker (2017) the same time step is used for both the low-resolution and the high-resolution ensemble forecasts because the GFS employs an unconditionally stable semi-Lagrangian time integration scheme which allows stable integration with long time steps (Williamson 2007). The cost of the 4DEnVar update is sensitive to the resolution of the extended control variable which has the same dimension as the background ensemble as shown in Eq. (1). The cost of each method is summarized in Table 2. Because EnKF is conducted at the low-resolution (T254) for all experiments, all the experiments have the same cost of the EnKF update as shown in the third column of Table 2. The last column shows the ratio of the cost relative to the SR-Low experiment. These results are broadly consistent with the theoretical estimation. The computational cost of SR-High is about 4.63 times of SR-Low. In comparison, the cost of MR-ENS is about 2.81 times of the SR-Low, $40 \%$ lower than SRHigh. As shown in sections $4 \mathrm{~b}$ and $4 \mathrm{c}$, MR-ENS shows comparable skill in terms of global forecast and tropical cyclone track forecast with SR-High, suggesting MR-ENS is a more cost-efficient approach than SR-High. Finally, the cost of DR is $21 \%$ higher than SR-Low due to the additional computation of using the high-resolution control background and high-resolution static covariance during the 4DEnVar minimization.

\section{Summary and discussion}

Due to the constraints of limited computational resources, the background ensemble is run at a lower resolution than the control forecast in the operational GSI-based 4DEnVar in the global NWP system at NCEP. In this study, the GSI-based 4DEnVar is further developed to have the capability to ingest ensembles at multiple resolutions (MR-ENS). Therefore, effective ensemble covariance resolving different ranges of scales are used in the analysis update. Compared to the approach where all members are at high resolution, MR-ENS offers an inexpensive means to increase the resolution of the flow-dependent BEC. The effect of the resolutions of the ensemble BEC on the analysis and subsequent forecast is investigated by conducting the following experiments: DR using high-resolution control background, high-resolution static BEC and low-resolution ensemble BEC, newly developed MR-ENS, which is the same as DR except using partial high- and partial low-resolution ensemble BECs, SR-High where all components including the control background, ensemble BEC and static BEC are at a single high resolution. MR-ENS is further compared with SR-Low which is similar to the operational configuration where both the flowdependent BEC and static BEC are at a single low resolution, and the control background is interpolated to the same low resolution during the analysis increment calculation.

Overall MR-ENS improves the global forecast for 15-day lead times compared to SR-Low and DR. It also improves tropical cyclone track forecast out to $\sim 5$ - and $\sim 3$-day lead times compared to SR-Low and DR, respectively. Diagnostics have performed to further explore this result. The inclusion of high-resolution ensembles through MR-ENS increases the background ensemble spread and reduces the RMS error of the background. The observation term of the cost function of MR-ENS fits the observations more closely. Though the same number of ensemble members is used, the effective rank of the ensemble BEC of MR-ENS is higher than that of DR and SR-Low. Scale-dependent diagnostics of the analysis increment shows that the high-resolution ensemble BEC contributes to the analysis increment of the small scales much more than DR and SR-Low, due to better representation of flow-dependent error covariances for a wider range of scales. Additional Wavelet diagnostics show MR-ENS has larger increments for small scales in convectively active regions over the tropics. The verification of ensemble estimated background error correlation shows that MR-ENS improves the autocorrelation for both the large and small scales for $\mathrm{NH}$ and $\mathrm{SH}$ and improves the autocorrelation for small-scales in TR. Last, except for the tropical cyclone 
forecasts, the improvement of MR-ENS relative to SRLow is mostly contributed by the partial use of the high-resolution background ensemble.

Although MR-ENS requires $60 \%$ of the cost of SRHigh, MR-ENS shows comparable skill for the global and tropical cyclone track forecasts compared to SRHigh. Diagnostics shows that MR-ENS shows slightly less background ensemble spread and slightly less fit of the background to observations for the wind field. The background ensemble from MR-ENS shows less effective rank in NH and SH, but comparable rank in TR compared to SR-High. Scale-dependent evaluation of the analysis increment shows that the difference between MR-ENS and SR-High at small scales is much smaller than their differences from DR or SR-Low. Overall the results above suggest MR-ENS is a more cost-efficient way to use the ensemble to better estimate the background error covariance of a wider range of scales.

This study is among the first to explore the multiresolution ensemble approach in 4DEnVar in a real global NWP context. As an initial investigation, experiments are primarily designed or limited to focus on addressing the impact of the resolution of the ensemble BECs for a given total number of background ensemble members. In addition, the new MR-ENS experiment adopted the same number of high and low-resolution ensemble members. As shown in Eqs. (4) and (5) and discussed in section 2b, the MR-ENS 4DEnVar formula allows the low- and high-resolution ensemble members to use different ensemble size and different covariance localization. Further studies using the system developed are planned to address the optimal multiresolution and multiscale data assimilation under the constraints of the same computational cost. The optimal weighting of the static and ensemble covariances in the context of multiresolution and multiscale data assimilation should be also explored in the future. In the current study, the MR-ENS method is applied to the 4DEnVar method. The same approach can also be applied in various other types of hybrid DA method (e.g., Lorenc 2003; Wang and Lei 2014; Zhang et al. 2013; Poterjoy and Zhang 2016). As MR-ENS can resolve a wider range of scales in the background error covariance without incurring too much additional cost, this approach is particularly appealing in the operational NWP which is subject to computational resource constraint. MR-ENS saves the cost by only running partial ensemble members at high resolution. The cost can be even reduced by using the valid time shifting approach to populate the highresolution members following Huang and Wang (2018).

Acknowledgments. The study is supported by NOAA NGGPS Award NA15NWS4680022. The NOAA High
Performance Computing System Jet machine and the OU Supercomputing Center for Education and Research at the University of Oklahoma were provided for the computational resources for this study. The authors thank NOAA collaborators, in particular, Rahul Mahajan, Daryl Kleist, Ting Lei, Lili Lei, and Jeff Whitaker, for providing needed observations and scripts. The authors also thank Bo Huang for helpful discussion. Some results and discussion were presented at the AMS conference.

\section{APPENDIX}

\section{Implementation of Multiresolution Ensemble (MR-ENS) 4DEnVar in GSI}

In this appendix, the mathematical formula describing the specific implementation of the Multiresolution ensemble (MR-ENS) 4DEnVar in the GSI minimization is included. The key of this derivation is to demonstrate that the minimization of the cost function of MR-ENS with two sets of extended control variables can fit the full background error covariance preconditioning of the original GSI 4DEnVar minimization (Wang 2010; Wang and Lei 2014). The demonstration follows and extends from Wang (2010) and Wang and Lei (2014) which describe the mathematical formula on how to implement 3D and 4D EnVar in GSI.

The analysis increment and cost function of MR-ENS are defined as Eqs. (4) and (5) in the main text. The extended control variable used in minimization is defined as

$$
\mathbf{x}=\left(\begin{array}{c}
\mathbf{x}_{1}^{\prime} \\
\boldsymbol{\alpha}^{L} \\
\boldsymbol{\alpha}^{H}
\end{array}\right)
$$

The analysis increment [Eq. (4)] can alternatively be expressed as

$$
\begin{aligned}
\mathbf{x}^{\prime}= & \mathbf{x}_{1}^{\prime}+\mathbf{u} \sum_{k=1}^{K_{L}}\left(\boldsymbol{\alpha}_{k}^{L} \circ \mathbf{x}_{k}^{e L}\right)+\sum_{k=1}^{K_{H}}\left(\boldsymbol{\alpha}_{k}^{H} \circ \mathbf{x}_{k}^{e H}\right) \\
= & \mathbf{x}_{1}^{\prime}+\mathbf{u}\left[\operatorname{diag}\left(\mathbf{x}_{1}^{e L}\right), \ldots, \operatorname{diag}\left(\mathbf{x}_{K_{L}}^{e L}\right)\right] \boldsymbol{\alpha}^{L} \\
& +\left[\operatorname{diag}\left(\mathbf{x}_{1}^{e H}\right), \ldots, \operatorname{diag}\left(\mathbf{x}_{K_{H}}^{e H}\right)\right] \boldsymbol{\alpha}^{H}
\end{aligned}
$$

or

$$
\mathbf{x}^{\prime}=\mathbf{x}_{1}^{\prime}+\mathbf{u D}_{L} \boldsymbol{\alpha}^{L}+\mathbf{D}_{H} \boldsymbol{\alpha}^{H}=\mathbf{C} \mathbf{x},
$$

where $\mathbf{D}_{L}=\operatorname{diag}\left(\mathbf{x}_{1}^{e L}\right), \ldots, \operatorname{diag}\left(\mathbf{x}_{K}^{e L}\right), \mathbf{D}_{H}=\operatorname{diag}\left(\mathbf{x}_{1}^{e H}\right), \ldots$, $\operatorname{diag}\left(\mathbf{x}_{K}^{e H}\right)$, and $\mathbf{C}=\left(\mathbf{I}, \mathbf{u} \mathbf{D}_{L}, \mathbf{D}_{H}\right)$. Further denote the new background error covariance as 


$$
\mathbf{B}=\left(\begin{array}{ccc}
\frac{1}{\beta_{1}} \mathbf{B}_{1} & 0 & 0 \\
0 & \frac{1}{\beta_{L}} \mathbf{A}_{L} & 0 \\
0 & 0 & \frac{1}{\beta_{H}} \mathbf{A}_{H}
\end{array}\right) .
$$

As in the original GSI, the preconditioned new control variable $\mathbf{z}$ is defined as

$$
\mathbf{z}=\mathbf{B}^{-1} \mathbf{x}=\left(\begin{array}{ccc}
\beta_{1} \mathbf{B}_{1}^{-1} & 0 & 0 \\
0 & \beta_{L} \mathbf{A}_{L}^{-1} & 0 \\
0 & 0 & \beta_{H} \mathbf{A}_{H}^{-1}
\end{array}\right)\left(\begin{array}{c}
\mathbf{x}_{1}^{\prime} \\
\boldsymbol{\alpha}^{L} \\
\boldsymbol{\alpha}^{H}
\end{array}\right) .
$$

As discussed in Wang (2010) and Wang and Lei (2014), if we explicitly show $\nabla_{\mathbf{z}} J$ and $\nabla_{\mathbf{x}} J$ with respect to the control variables associated with the static covariance and with respect to the two sets of extended control variables associated with the high and low resolution ensembles, and demonstrate $\nabla_{\mathbf{z}} J=\mathbf{B} \nabla_{\mathbf{x}} J$, then the minimization for the multiresolution ensemble 4DEnVar cost function can simply follow the same conjugate gradient method by the original GSI. Gradient of $J$ with respect to $\mathbf{x}, \nabla_{\mathbf{x}} J$, is defined as

$$
\begin{aligned}
& \nabla_{\mathbf{x}_{1}^{\prime}} J=\beta_{1} \mathbf{B}_{1}^{-1} \mathbf{x}_{1}^{\prime}+\mathbf{H}^{\mathrm{T}} \mathbf{R}^{-1}\left(\mathbf{H} \mathbf{x}^{\prime}-\mathbf{y}^{o}\right), \\
& \nabla_{\boldsymbol{\alpha}^{L}} J=\beta_{L} \mathbf{A}_{L}^{-1} \boldsymbol{\alpha}^{L}+\beta_{L} \mathbf{A}_{L}^{-1} \boldsymbol{\alpha}^{L}+\mathbf{D}_{\mathrm{L}}^{\mathrm{T}} \mathbf{u}^{\mathrm{T}} \mathbf{H}^{\mathrm{T}} \mathbf{R}^{-1}\left(\mathbf{H} \mathbf{x}^{\prime}-\mathbf{y}^{o}\right),
\end{aligned}
$$

$\nabla_{\boldsymbol{\alpha}^{H}} J=\beta_{H} \mathbf{A}_{H}^{-1} \boldsymbol{\alpha}^{H}+\mathbf{D}_{H}^{\mathrm{T}} \mathbf{H}^{\mathrm{T}} \mathbf{R}^{-1}\left(\mathbf{H} \mathbf{x}^{\prime}-\mathbf{y}^{o}\right)$.

Therefore, using Eqs. (A3)-(A8), we obtain

$$
\begin{aligned}
\nabla_{\mathbf{x}} J=\left(\begin{array}{c}
\nabla_{\mathbf{x}_{1}^{\prime}} J \\
\nabla_{\boldsymbol{\alpha}^{L}} J \\
\nabla_{\boldsymbol{\alpha}^{H}} J
\end{array}\right) & =\mathbf{B}^{-1} \mathbf{x}+\mathbf{C}^{\mathrm{T}} \mathbf{H}^{\mathrm{T}} \mathbf{R}^{-1}\left(\mathbf{H} \mathbf{x}^{\prime}-\mathbf{y}^{o}\right) \\
& =\mathbf{z}+\mathbf{C}^{\mathrm{T}} \mathbf{H}^{\mathrm{T}} \mathbf{R}^{-1}\left(\mathbf{H}^{\prime}-\mathbf{y}^{o}\right) .
\end{aligned}
$$

The gradient of $J$ with respect to $\mathbf{z}, \nabla_{\mathbf{z}} J$, is defined as

$$
\begin{aligned}
\nabla_{\beta_{1} \mathbf{B}_{1}^{-1} \mathbf{x}_{1}^{\prime}} J & =\mathbf{x}_{1}^{\prime}+\frac{1}{\beta_{1}} \mathbf{B}_{1} \mathbf{H}^{\mathrm{T}} \mathbf{R}^{-1}\left(\mathbf{H} \mathbf{x}^{\prime}-\mathbf{y}^{o}\right), \\
\nabla_{\beta_{L} \mathbf{A}_{L}^{-1} \boldsymbol{\alpha}^{L}} J & =\boldsymbol{\alpha}^{L}+\frac{1}{\beta_{L}} \mathbf{A}_{L} \mathbf{D}_{L}^{\mathrm{T}} \mathbf{u}^{\mathrm{T}} \mathbf{H}^{\mathrm{T}} \mathbf{R}^{-1}\left(\mathbf{H} \mathbf{x}^{\prime}-\mathbf{y}^{o}\right), \\
\nabla_{\beta_{H} \mathbf{A}_{H}^{-1} \boldsymbol{\alpha}^{H}} J & =\boldsymbol{\alpha}^{H}+\frac{1}{\beta_{H}} \mathbf{A}_{H} \mathbf{D}_{H}^{\mathrm{T}} \mathbf{H}^{\mathrm{T}} \mathbf{R}^{-1}\left(\mathbf{R x}^{\prime}-\mathbf{y}^{o}\right) .
\end{aligned}
$$

Using Eqs. (A3)-(A5) and (A10)-(A12), we obtain

$$
\nabla_{\mathbf{z}} J=\left(\begin{array}{c}
\nabla_{\beta_{1} \mathbf{B}_{1}^{-1} \mathbf{x}_{1}^{\prime} J} \\
\nabla_{\beta_{L} \mathbf{A}_{L}^{-1} \boldsymbol{\alpha}^{L} J} J \\
\nabla_{\beta_{H} \mathbf{A}_{H}^{-1} \boldsymbol{\alpha}^{H} J} J
\end{array}\right)=\mathbf{x}+\mathbf{B C}^{\mathrm{T}} \mathbf{H}^{\mathrm{T}} \mathbf{R}^{-1}\left(\mathbf{H} \mathbf{x}^{\prime}-\mathbf{y}^{o}\right) .
$$

Comparing $\nabla_{\mathrm{x}} J$ in Eq. (A9) and $\nabla_{\mathrm{z}} J$ in Eq. (A13), we thus obtain

$$
\begin{gathered}
\nabla_{\mathbf{z}} J=\mathbf{B} \nabla_{\mathbf{x}} J . \\
\text { REFERENCES }
\end{gathered}
$$

Aligo, E. A., W. A. Gallus Jr., and M. Segal, 2009: On the impact of WRF model vertical grid resolution on Midwest summer rainfall forecasts. Wea. Forecasting, 24, 575-594, https:// doi.org/10.1175/2008WAF2007101.1.

Baklanov, A. A., and Coauthors, 2011: The nature, theory, and modeling of atmospheric planetary boundary layers. Bull. Amer. Meteor. Soc., 92, 123-128, https://doi.org/10.1175/ 2010BAMS2797.1.

Balle, T., and P. Johnsen, 2016: Improving I/O performance of the Weather Research and Forecasting (WRF) model. Scalability CUG 2016, London, United Kingdom, ECMWF, 9 pp., https://cug.org/proceedings/cug2016_proceedings/includes/ files/pap123s2-file1.pdf.

Berner, J., M. L. G. Shutts, and T. Palmer, 2009: A spectral stochastic kinetic energy backscatter scheme and its impact on flow dependent predictability in the ECMWF ensemble prediction system. J. Atmos. Sci., 66, 603-626, https://doi.org/ 10.1175/2008JAS2677.1.

Bloomfield, P., 2002: Fourier Analysis of Time Series: An Introduction. 2nd ed. Wiley, 288 pp.

Bonavita, M., L. Raynaud, and L. Isaksen, 2011: Estimating background-error variances with the ECMWF Ensemble of Data Assimilations system: Some effects of ensemble size and day-to-day variability. Quart. J. Roy. Meteor. Soc., 137, 423434, https://doi.org/10.1002/qj.756.

Buehner, M., and A. Shlyaeva, 2015: Scale-dependent backgrounderror covariance localisation. Tellus, 67A, 28027, https:// doi.org/10.3402/tellusa.v67.28027.

Caron, J., and M. Buehner, 2018: Scale-dependent background error covariance localization: Evaluation in a global deterministic weather forecasting system. Mon. Wea. Rev., 146, 1367-1381, https://doi.org/10.1175/MWR-D-17-0369.1.

Clayton, A. M., A. C. Lorenc, and D. M. Barker, 2013: Operational implementation of a hybrid ensemble/4D-Var global data assimilation system at the Met Office. Quart. J. Roy. Meteor. Soc., 139, 1445-1461, https://doi.org/10.1002/qj.2054.

Daley, R., 1994: Atmospheric Data Assimilation. Cambridge University Press, 457 pp.

Domingues, M. O., O. Mendes, and A. M. da Costa, 2005: On wavelet techniques in atmospheric sciences. Adv. Space Res., 35, 831-842, https://doi.org/10.1016/j.asr.2005.02.097.

Evans, J. L., J. D. Fuentes, X.-M. Hu, and H. Hamilton, 2011: Earth-atmosphere interactions: Tropical storm and hurricane activity in the Caribbean and their consequent health impacts. J. Race Policy, 7 (1), 53-74. 
Gaspari, G., and S. E. Cohn, 1999: Construction of correlation functions in two and three dimensions. Quart. J. Roy. Meteor. Soc., 125, 723-757, https://doi.org/10.1002/qj.49712555417.

Hamill, T. M., J. S. Whitaker, M. Fiorino, S. E. Koch, and S. J. Lord, 2010: Increasing NOAA's computational capacity to improve global forecast modeling: A NOAA white paper. NOAA, 25 pp., https://www.esrl.noaa.gov/psd/people/ tom.hamill/global_HPC_whitepaper_hamilletal.pdf.

Hayden, C. M., and R. J. Purser, 1995: Recursive filter objective analysis of meteorological fields: applications to NESDIS operational processing. J. Appl. Meteor., 34, 3-15, https://doi.org/ 10.1175/1520-0450-34.1.3.

Holton, J. R., 2004: An Introduction to Dynamic Meteorology. 4th ed. Academic Press, 535 pp.

Houtekamer, P. L., and H. L. Mitchell, 2001: A sequential ensemble Kalman filter for atmospheric data assimilation. Mon. Wea. Rev., 129, 123-137, https://doi.org/10.1175/1520-0493(2001)129<0123: ASEKFF $>2.0 . C O ; 2$.

Huang, B., and X. Wang, 2018: On the use of cost-effective validtime-shifting (VTS) method to increase ensemble size in the GFS Hybrid 4DEnVar system. Mon. Wea. Rev., 146, 2973 2998, https://doi.org/10.1175/MWR-D-18-0009.1.

Janjić, T., and Coauthors, 2018: On the representation error in data assimilation. Quart. J. Roy. Meteor. Soc., 144, 1257-1278, https://doi.org/10.1002/qj.3130.

Johnson, A., and Coauthors, 2014: Multiscale characteristics and evolution of Perturbations for warm season convectionallowing precipitation forecasts: Dependence on background flow and method of perturbation. Mon. Wea. Rev., 142, 10531073, https://doi.org/10.1175/MWR-D-13-00204.1.

,,- K. R. Haghi, and D. B. Parsons, 2018: Evaluation of forecasts of a convectively generated bore using an intensively observed case study from PECAN. Mon. Wea. Rev., 146, 30973122, https://doi.org/10.1175/MWR-D-18-0059.1.

Kay, J. K., and H. M. Kim, 2014: Characteristics of initial perturbations in the ensemble prediction system of the Korea Meteorological Administration. Wea. Forecasting, 29, 563581, https://doi.org/10.1175/WAF-D-13-00097.1.

Kim, S.-M., and H. M. Kim, 2014: Sampling error of observation impact statistics. Tellus, 66A, 25435, https://doi.org/10.3402/ tellusa.v66.25435.

Kleist, D. T., 2012: An evaluation of hybrid variational-ensemble data assimilation for the NCEP GFS. Ph.D. thesis, Dept. of Atmospheric and Oceanic Science, University of Maryland, College Park, College Park, MD, 149 pp.

—_, and K. Ide, 2015: An OSSE-based evaluation of hybrid variational-ensemble data assimilation for the NCEP GFS. Part I: System description and 3D-hybrid results. Mon. Wea. Rev., 143, 433-451, https://doi.org/10.1175/ MWR-D-13-00351.1.

Kuhl, D. D., and Coauthors, 2007: Assessing predictability with a local ensemble Kalman filter. J. Atmos. Sci., 64, 1116-1140, https://doi.org/10.1175/JAS3885.1.

Lei, L., and J. S. Whitaker, 2017: Evaluating the trade-offs between ensemble size and ensemble resolution in an ensemblevariational data assimilation system. J. Adv. Model. Earth Syst., 9, 781-789, https://doi.org/10.1002/2016MS000864.

Liebmann, B., and C. A. Smith, 1996: Description of a complete (interpolated) outgoing longwave radiation dataset. Bull. Amer. Meteor. Soc., 77, 1275-1277.

Liu, H., F. Sassi, and R. R. Garcia, 2009: Error growth in a whole atmosphere climate model. J. Atmos. Sci., 66, 173-186, https:// doi.org/10.1175/2008JAS2825.1.
Lorenc, A. C., 2003: The potential of the ensemble Kalman filter for NWP-A comparison with 4D-Var. Quart. J. Roy. Meteor. Soc., 129, 3183-3203, https://doi.org/10.1256/qj.02.132.

Lu, X., X. Wang, M. Tong, and V. Tallapragada, 2017: GSI-based, continuously cycled, dual-resolution hybrid ensemblevariational data assimilation system for HWRF: System description and experiments with Edouard (2014). Mon. Wea. Rev., 145, 4877-4898, https://doi.org/10.1175/ MWR-D-17-0068.1.

Ma, J., Y. Zhu, R. Wobus, and P. Wang, 2012: An effective configuration of ensemble size and horizontal resolution for the NCEP GEFS. Adv. Atmos. Sci., 29, 782-794, https://doi.org/ 10.1007/s00376-012-1249-y.

Marchok, T. P., 2002: How the NCEP tropical cyclone tracker works. Preprints, 25th Conf. on Hurricanes and Tropical Meteorology, San Diego, CA, Amer. Meteor. Soc., P1.13, http://ams.confex.com/ams/pdfpapers/37628.pdf.

Meng, Z., and F. Zhang, 2007: Tests of an ensemble Kalman filter for mesoscale and regional-scale data assimilation. Part II: Imperfect model experiments. Mon. Wea. Rev., 135, 14031423, https://doi.org/10.1175/MWR3352.1.

Palmer, T. N., R. Buizza, F. Doblas-Reyes, T. Jung, M. Leutbecher, G. J. Shutts, M. Steinheimer, and A. Weisheimer, 2009: Stochastic parametrization and model uncertainty. ECMWF Tech. Memo. 598, 44 pp., https://www.ecmwf.int/en/elibrary/ 11577-stochastic-parametrization-and-model-uncertainty.

Patil, D. J., B. R. Hunt, E. Kalnay, J. A. Yorke, and E. Ott, 2001: Local low dimensionality of atmospheric dynamics. Phys. Rev. Lett., 86, 5878-5881, https://doi.org/10.1103/ PhysRevLett.86.5878.

Poterjoy, J., and F. Zhang, 2016: Comparison of hybrid fourdimensional data assimilation methods with and without the tangent linear and adjoint models for predicting the life cycle of Hurricane Karl (2010). Mon. Wea. Rev., 144, 1449-1468, https://doi.org/10.1175/MWR-D-15-0116.1.

Rainwater, S., and B. Hunt, 2013: Mixed-resolution ensemble data assimilation. Mon. Wea. Rev., 141, 3007-3021, https://doi.org/ 10.1175/MWR-D-12-00234.1.

Raynaud, L., and F. Bouttier, 2017: The impact of horizontal resolution and ensemble size for convective-scale probabilistic forecasts. Quart. J. Roy. Meteor. Soc., 143, 3037-3047, https:// doi.org/10.1002/qj.3159.

Reichler, T., and J. O. Roads, 2005: Long-range predictability in the tropics. Part I: Monthly averages. J. Climate, 18, 619-633, https://doi.org/10.1175/JCLI-3294.1.

Steeneveld, G. J., B. J. H. van de Wiel, and A. A. M. Holtslag, 2006: Modelling the arctic stable boundary layer and its coupling to the surface. Bound.-Layer Meteor., 118, 357-378, https:// doi.org/10.1007/s10546-005-7771-z.

Straus, D., and D. Paolino, 2008: Intermediate time error growth and predictability: Tropics versus mid-latitudes. Tellus, $\mathbf{6 1 A}$, 579-586, https://doi.org/10.1111/j.1600-0870.2009.00411.x.

Tompkins, A. M., and J. Berner, 2008: A stochastic convective approach to account for model uncertainty due to unresolved humidity variability. J. Geophys. Res., 113, D18101, https:// doi.org/10.1029/2007JD009284.

Torrence, C., and G. P. Compo, 1998: A practical guide to wavelet analysis. Bull. Amer. Meteor. Soc., 79, 61-78, https://doi.org/ 10.1175/1520-0477(1998)079<0061:APGTWA > 2.0.CO;2.

Wang, X., 2010: Incorporating ensemble covariance in the gridpoint statistical interpolation variational minimization: A mathematical framework. Mon. Wea. Rev., 138, 2990-2995, https://doi.org/10.1175/2010MWR3245.1. 
- and C. H. Bishop, 2003: A comparison of breeding and ensemble transform Kalman filter ensemble forecast schemes. J. Atmos. Sci., 60, 1140-1158, https://doi.org/10.1175/15200469(2003)060<1140:ACOBAE > 2.0.CO;2.

_ , and T. Lei, 2014: GSI-based four-dimensional ensemblevariational (4DEnsVar) data assimilation: Formulation and single-resolution experiments with real data for NCEP Global Forecast System. Mon. Wea. Rev., 142, 3303-3325, https:// doi.org/10.1175/MWR-D-13-00303.1.

, C. Snyder, and T. M. Hamill, 2007: On the theoretical equivalence of differently proposed ensemble-3DVAR hy brid analysis schemes. Mon. Wea. Rev., 135, 222-227, https:// doi.org/10.1175/MWR3282.1.

_ _ D. Parrish, D. Kleist, and J. Whitaker, 2013: GSI 3DVarbased ensemble-variational hybrid data assimilation for NCEP Global Forecast System: Single-resolution experiments. Mon. Wea. Rev., 141, 4098-4117, https://doi.org/ 10.1175/MWR-D-12-00141.1.

Whitaker, J. S., and T. M. Hamill, 2012: Evaluating methods to account for system errors in ensemble data assimilation. Mon. Wea. Rev., 140, 3078-3089, https://doi.org/10.1175/MWR-D11-00276.1.
X. Wei, Y. Song, and Z. Toth, 2008: Ensemble data assimilation with the NCEP Global Forecast System. Mon. Wea. Rev., 136, 463-482, https://doi.org/10.1175/2007MWR2018.1.

Wilks, D. S., 2006: Statistical Methods in the Atmospheric Sciences. 2nd ed. International Geophysics Series, Vol. 100, Academic Press, 648 pp.

Williamson, D. L., 2007: The evolution of dynamical cores for global atmospheric models. J. Meteor. Soc. Japan, 85, 241-269, https://doi.org/10.2151/jmsj.85B.241.

Yang, E., and H. M. Kim, 2017: Evaluation of a regional reanalysis and ERA-Interim over East Asia using in situ observations during 2013-14. J. Appl. Meteor. Climatol., 56, 2821-2844, https://doi.org/10.1175/JAMC-D-16-0227.1.

Zhang, F., Z. Meng, and A. Aksoy, 2006: Tests of an ensemble Kalman filter for mesoscale and regional-scale data assimilation. Part I: Perfect model experiments. Mon. Wea. Rev., 134, 722-736, https://doi.org/10.1175/MWR3101.1.

- - M. Zhang, and J. Poterjoy, 2013: E3DVar: Coupling an ensemble Kalman filter with three-dimensional variational data assimilation in a limited-area weather prediction model and comparison to E4DVar. Mon. Wea. Rev., 141, 900-917, https://doi.org/10.1175/MWR-D-12-00075.1. 